\title{
PAPR Reduction Approach Based on Channel Estimation Pilots for Next Generations Broadcasting Systems
}

\author{
Anh-Tai Ho, ${ }^{1}$ Jean-François Helard, ${ }^{1}$ Youssef Nasser, ${ }^{2}$ and Yves Louet ${ }^{3}$ \\ ${ }^{1}$ IETR UMR 6164, INSA, Université Européenne de Bretagne, 35708 Rennes, France \\ ${ }^{2}$ American University of Beirut, Bliss Street, Beirut 11-0236, Lebanon \\ ${ }^{3}$ SCEE Team, Supelec, avenue de la Boulaie, 35576 Cesson-Sévigné, France
}

Correspondence should be addressed to Youssef Nasser, youssef.nasser@aub.edu.lb

Received 12 October 2010; Accepted 4 January 2011

Academic Editor: Massimiliano Laddomada

Copyright (c) 2011 Anh-Tai Ho et al. This is an open access article distributed under the Creative Commons Attribution License, which permits unrestricted use, distribution, and reproduction in any medium, provided the original work is properly cited.

\begin{abstract}
A novel peak-to-average power ratio (PAPR) reduction technique for orthogonal frequency division multiplexing (OFDM) systems is addressed. Instead of using dedicated pilots for PAPR reduction as with tone reservation (TR) method selected by the DVB-T2 standard, we propose to use existing pilots used for channel estimation. In this way, we avoid the use of reserved tone pilots and then improve the spectral efficiency of the system. In order to allow their recovery at the receiver, these pilots have to follow particular laws which permit their blind detection and avoid sending side information. In this work, we propose and investigate a multiplicative law operating in discrete frequency domain. The operation in discrete domain aims at reducing degradation due to detection and estimation error in continuous domain. Simulation results are performed using the new DVB-T2 standard parameters. Its performance is compared to the DVB-T2 PAPR gradient algorithm and to the second-order cone programming (SOCP) competitive technique proposed in the literature. We show that the proposed technique is efficient in terms of PAPR reduction value and of spectral efficiency while the channel estimation performance is maintained.
\end{abstract}

\section{Introduction}

orthogonal frequency division multiplexing (OFDM) technology has been the subject of numerous dissertations in recent years, mainly due to its several advantages for mobile wireless communications. It has been adopted in several systems mainly in digital video broadcasting (DVB) standards [1-3].

However, a main drawback of OFDM technique is the high peak-to-average power ratio (PAPR) of the transmitted signal. High PAPR value implies sophisticated and, thus, expensive radio transmitters with high-power amplifiers (HPAs) operating on a very large linear range at the transmitter side. Moreover, the nonlinearity of the HPA leads to in-band distortion, which increases the bit error rate (BER) of the system, and to out-of-band (OOB) distortion, which introduces high adjacent channel interference.

Various approaches have been proposed as summarized in $[4,5]$ to mitigate the PAPR of an OFDM signal. Among them, clipping and filtering technique is easy to implement.
However, these schemes yield a broken system performance since clipping is a nonlinear process [6] leading to a signal distortion. An alternative method based on coding was proposed in [7], in which a data sequence is embedded in a longer sequence, and only a subset of all these possible sequences is used to exclude patterns generating high PAPR. Moreover, other methods such as partial transmit sequence [8], selected mapping [9], and interleaving [10] are also proposed. The main drawback of these methods is the necessity to transmit side information (SI) to the receiver, resulting in some loss of throughput efficiency.

Some methods recently proposed do not need this SI transmission $[11,12]$. Indeed, the active constellation extension (ACE) method proposed in [12] reduces the PAPR by changing the constellation of the signal without modifying the minimum distance. However, this technique has some gain limits when the constellation size of the signal increases. Moreover, this technique is not suited for rotated constellation schemes, which have been selected for the second generation digital terrestrial television broadcasting system 
(DVB-T2). The tone reservation (TR) method, proposed by Tellado and Tellambura [10] and adopted in DVB-T2 also, uses allocated subcarriers to generate additional signal that minimizes the PAPR value. However, TR technique reduces the spectrum efficiency since it requires some dedicated pilots for PAPR reduction issues. In DVB-T2, $1 \%$ of the subcarriers are dedicated for PAPR. Moreover, an iterative process should be implemented at the transmitter with a special need for a smooth control of the transmitted power on the dedicated subcarriers in order to respect the DVBT2 spectrum mask requirements (the dedicated pilots should be at a power level less than $10 \mathrm{~dB}$ with respect to the data subcarriers power).

With the limitations of ACE technique for rotated constellation schemes and those of TR by spectral efficiency loss, an innovative technique which could be implemented for rotated constellation and without efficiency loss is clearly needed. In this paper, we adopt, as in [4], the TR technique by using the pilots of channel estimation for both PAPR reduction and channel estimation issues. The novelty of our proposition is based on the optimization of the phase and amplitude of the channel estimation pilots, used jointly for PAPR reduction process. Indeed, instead of using orthogonal pilots sequences (OPSs), we optimize the transmitted sequence for PAPR issue in terms of phase and amplitude. Using a predefined law between different sequences, the receiver could easily utilize a blind detection of the transmitted sequences.

The proposition of this work is multifold. First, instead of using dedicated pilots for PAPR reduction, we expend the idea of [4] which consists in utilizing existing pilots dedicated for channel estimation for both channel estimation and PAPR reduction issues. In this way, we avoid the use of reserved pilots as proposed in DVB-T2 standard improving thus the spectral efficiency of the system. Second, in order to allow their recovery serving for channel estimation process at the receiver, these pilots have to follow particular laws. Multiplicative law in frequency domain is then proposed and investigated in this work. At the receiver, this law is applied to detect and estimate transmitted pilots in frequency domain. As the detection and estimation of multiplicative law's parameters in continuous frequency domain, that is, in real space, cause degradations, we propose to operate this law in the discrete frequency domain, that is, in a predefined discrete set. Third, we show by simulations the validity of our technique using DVB-T2 standard [1] parameters. Its performance is compared to the DVB-T2 PAPR gradient algorithm and to the secondorder cone programming (SOCP) technique proposed in [13-15].

The remainder of this paper is organized as follows. Section 2 briefly presents the OFDM system model and the PAPR statistics. Then, in order to have a clear overview about the benefits of our proposed technique, we will first explain the general principle of the TR-based techniques in Section 3. In Section 4, we describe our proposed pilot-aided PAPR reduction technique. In Section 5, blind pilot sequence detection and channel estimation are introduced. Section 6 presents the performance of the proposed method as well as a summary of the obtained results. Finally, conclusions are drawn in Section 7.

\section{OFDM Signal and PAPR Value}

Let $\mathbf{X}=\left[X_{0}, \ldots, X_{N-1}\right]$ be a sequence of complex symbols and $\mathbf{x}=\left[x_{0}, \ldots, x_{N-1}\right]$ be its discrete inverse Fourier transform. The OFDM baseband signal in continuous time domain, is expressed as,

$$
x(t)=\frac{1}{\sqrt{N}} \sum_{k=0}^{N-1} X_{k} e^{j 2 \pi k t / T N}, \quad 0 \leq t<N T,
$$

where $j=\sqrt{-1}, N$ denotes the number of subcarriers and $T$ is the original complex signal duration. In practice, only $N L$ equidistant samples of $x(t)$ are considered, where $L$ represents the oversampling factor [13] used to make the signal as close as possible to the continuous signal. The oversampled signal is then given by

$$
x_{[n / L]}=\frac{1}{\sqrt{N}} \sum_{k=0}^{N-1} X_{k} e^{j 2 \pi k n / L N}, \quad \forall n \in[0 \cdots N L-1] .
$$

When the oversampling factor $L$ is large enough, the PAPR value of the OFDM signal is defined as the ratio of its maximum power divided by its average power. It is expressed as [16]

$$
\operatorname{PAPR}\{x(t)\} \approx \operatorname{PAPR}\left(\mathbf{x}_{L}\right)=\frac{\max _{n}\left|x_{n / L}\right|^{2}}{E\left\{\left|x_{n / L}\right|^{2}\right\}},
$$

where $\mathbf{x}_{L}=\mathbf{Q}_{L} \mathbf{X}_{L}, \mathbf{X}_{L}$ is the zero-padded oversampled vector of $\mathbf{X}, E\{\}$ denotes expectation operation, and $\mathbf{Q}_{L}$ is the inverse discrete Fourier transform matrix of size $N L . \mathbf{Q}_{L}$ is given by

$$
\mathbf{Q}_{L}=\frac{1}{\sqrt{N}}\left[\begin{array}{cccc}
1 & 1 & & 1 \\
1 & \mathrm{e}^{(\mathcal{A}) 1 \cdot 1} & \cdots & \mathrm{e}^{(\mathcal{A}) 1 \cdot(N L-1)} \\
\vdots & \ddots & \vdots \\
1 & \mathrm{e}^{(\mathcal{A})(N L-1) \cdot 1} & \cdots & \mathrm{e}^{(\mathcal{A})(N L-1) \cdot(N L-1)}
\end{array}\right]
$$

where $\mathcal{A}$ denotes $j 2 \pi / N L$.

In this study, the PAPR performance is evaluated using the complementary cumulative distribution function (CCDF). It is defined by the probability that the PAPR value exceeds a given threshold $\gamma$. For $L=1$, it can be expressed as [17]

$$
\begin{aligned}
\operatorname{CCDF}_{\mathrm{PAPR}} & =\operatorname{Pr}\left[\operatorname{PAPR}\left(\mathbf{x}_{L}\right)>\gamma, L=1\right] \\
& \approx 1-\left(1-e^{-\gamma}\right)^{N} .
\end{aligned}
$$

It is also demonstrated in [17] that the real PAPR value can be approximated independently of $L$ when $L \geq 4$ by

$$
\begin{aligned}
\operatorname{CCDF}_{\operatorname{PAPR}\{x(t)\}} & =\operatorname{Pr}\left[\operatorname{PAPR}\left(\mathbf{x}_{L}\right)>\gamma, L \geq 4\right] \\
& \approx 1-\left(1-e^{-\gamma}\right)^{2.8 N} .
\end{aligned}
$$

In this paper, we consider $L=4$ and use this expression as a theoretical PAPR reference value of the original OFDM signal. 


\section{Existing TR-PAPR Reduction Methods}

3.1. General Principle. In an OFDM0-based system (like DVB standards), the main idea of the TR technique is to use reserved pilots in order to reduce the PAPR value at the input of the power amplifier of the time domain transmitted signal.

Let us consider $M$ as the number of channel estimation pilots used for PAPR reduction issue and $\mathbf{P}=\left[P_{0}, \ldots, P_{M-1}\right]$ as the $M$ pilot positions dedicated for PAPR reduction, and let $\mathbf{C}=\left[C_{0}, \ldots, C_{M-1}\right]$ be the set of $M$ pilots transmitted on these positions, as shown in Figure 1. Then, the $N$ modulated symbols $\left\{X_{k}\right\}_{k=0 \cdots N-1}$ of the OFDM symbol in frequency domain are expressed as:

$$
X_{k}= \begin{cases}C_{k} & \text { if } k \in \mathbf{P}, \\ S_{k} & \text { if not }\end{cases}
$$

where $S_{k}$ is the data symbol transmitted on subcarrier $k$ and $C_{k}$ is a pilot symbol used for PAPR reduction.

The OFDM baseband signal in time domain, after pilot insertion, becomes

$$
\mathbf{x}_{L}=\mathbf{Q}_{L} \mathbf{X}_{L}=\mathbf{Q}_{L}\left(\mathbf{C}+\mathbf{S}_{L}\right)=\mathbf{Z}_{L} \mathbf{C}+\mathbf{s}_{L}
$$

where $S_{L}$ is the data vector represented in frequency domain of size $N$ (where the values at the pilots positions are set to 0 ), and $\mathbf{s}_{L}$ is its time domain representation. $\mathbf{Z}_{L}$ is given by

$\mathbf{Z}_{L}=\frac{1}{\sqrt{N}}\left[\begin{array}{cccc}1 & 1 & \cdots & 1 \\ \mathrm{e}^{(\mathcal{A}) 1 \cdot p_{1}} & \mathrm{e}^{(\mathcal{A}) 1 \cdot p_{2}} & \cdots & \mathrm{e}^{(\mathscr{A}) 1 \cdot p_{M}} \\ \vdots & & \ddots & \vdots \\ \mathrm{e}^{(\mathcal{A})(N L-1) \cdot p_{1}} & \mathrm{e}^{(\mathcal{A})(N L-1) \cdot p_{2}} & \cdots & \mathrm{e}^{(\mathcal{A})(N L-1) \cdot p_{M}}\end{array}\right]$,

and $L$ holds for oversampling factor.

The added that signal $\mathbf{c}_{L}=\mathbf{Z}_{L} \mathbf{C}$ has to cope with PAPR values. In literature, different techniques have been proposed to optimize this signal. Among them, the SOCP and the gradient solutions are two of the most promising keys which have been extensively studied for optimization purposes. In the next section, we will give a general overview about these techniques. Performance of these techniques will be used as reference in comparison with our proposed technique.

3.2. SOCP Solution. The PAPR reduction problem can be formulated as follows: reducing PAPR value leads to the minimization of the maximum peak value of the combined signal $\left(\mathbf{s}_{L}+\mathbf{Z}_{L} \mathbf{C}\right)$ while keeping the average power constant [12]. Mathematically speaking, this problem can be written as

$$
\min _{\mathbf{C}} \max _{n}\left|\mathbf{s}_{L}+\mathbf{Z}_{n, L}^{\text {row }} \mathbf{C}\right|
$$

where $\mathbf{Z}_{n, L}^{\text {row }}$ denotes the $n$th row of $\mathbf{Z}_{L}$.

Equation (10) can be also rewritten as

$$
\min _{\mathbf{C}}\left\|\mathbf{s}_{L}+\mathbf{Z}_{n, L}^{\text {row }} \mathbf{C}\right\|_{\infty}
$$

where $\|\cdot\|$ denotes the standard uniform norm.
Since the set $\mathbf{C}$ of pilots is used for PAPR reduction, the problem turns out now to find $\mathbf{C}$ which minimizes the PAPR of the current transmitted OFDM symbol. In the continuous domain, that is, for $\mathbf{C} \subset \mathbb{C}^{M}$, this search is formulated as a convex optimization problem and solved using SOCP problem [13-15]. The optimization process can take the form of

$$
\begin{array}{ll}
\text { Minimize } & \beta \\
\text { subject to } & \left\|\mathbf{s}_{L}+\mathbf{Z}_{n, L}^{\text {row }} \mathbf{C}\right\| \leq \beta \\
& \forall 0 \leq n \leq N L-1 .
\end{array}
$$

It has been shown that this technique provides the optimized value in terms of PAPR reduction, but it presents a high calculation complexity [13-15]. In our study, we will use this technique as a comparison term with other techniques mainly with the proposed joint PAPR reduction and channel estimation scheme.

3.3. Gradient Iterative-Based Method. This method, adopted in DVB-T2 standard, is a suboptimal solution of the SOCP method. It is based on the gradient iterative method using the clipping process. In order to apply this technique, the technical specifications of DVB-T2 allowed 1\% of active subcarriers for PAPR reduction issues. The pilots signal used for PAPR, defined as a reference kernel signal, is given by

$$
p=\frac{N}{N_{\mathrm{TR}}} \mathbf{Q}_{L}\left[1_{\mathrm{TR}}\right]
$$

where $N_{\mathrm{TR}}$ denotes the number of reserved subcarriers, [ $\left.1_{\mathrm{TR}}\right]$ denotes the $(N, 1)$ vector having $N_{\mathrm{TR}}$ elements of ones at the positions corresponding to the reserved carriers and $(N-$ $N_{\mathrm{TR}}$ ) elements of zeros at the others. This reference signal presents a peak at the position 0 .

For each iteration, the peak position $k$ of the initial signal is detected. Reference signal is shifted of $k$ positions in order to allow the reduction of peak signal to a predefined clipping value $V_{\text {clip. }}$. The reduction principle is described in Figure 2.

The procedure of the PAPR reduction algorithm is given as follows.

Initialization. the initial values for peak reduction signal in time domain are set to zero:

$\mathbf{c}^{(0)}=[0 \cdots 0]^{T}$, where $\mathbf{c}^{(i)}$ denotes the vector of the peak reduction signal computed at $i$ th iteration.

\section{Iterations.}

(1) $i$ starts from 1 ;

(2) find the maximum magnitude of $\left(x+\mathbf{c}^{(i-1)}\right)$, noted $y_{i}$, and the corresponding sample index $k_{i}$;

(3) if $y_{i}<V_{\text {clip }}$, go to step (5);

if $y_{i}>V_{\text {clip }}$, clip the signal peak to this value;

update the vector of peak reduction signal $\mathbf{c}^{(i)}$; 


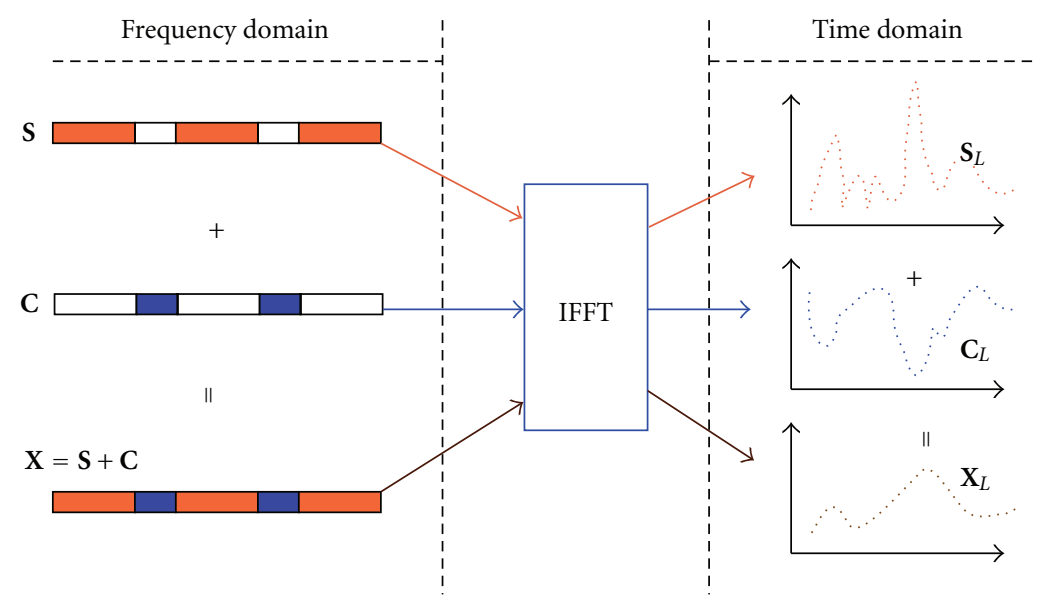

Figure 1: Pilots insertion scheme.

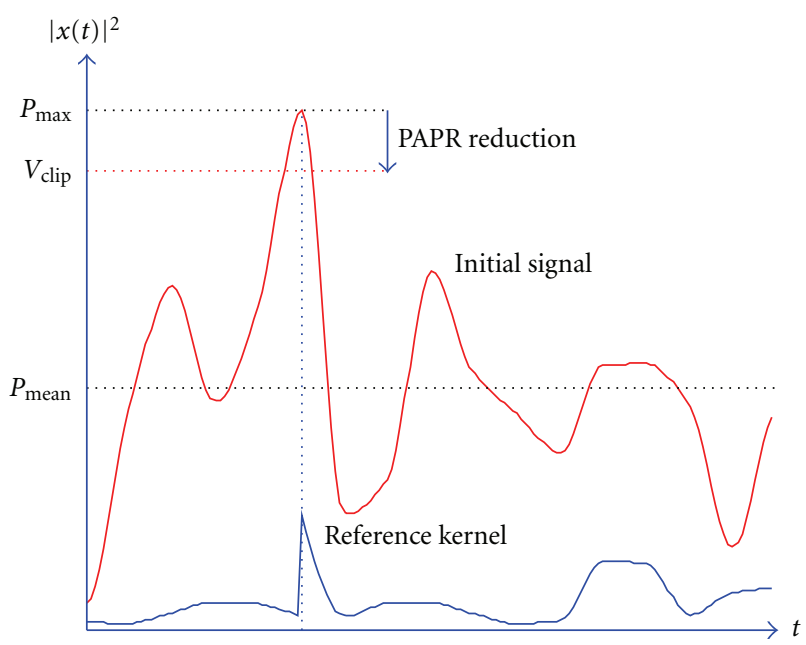

FIgURE 2: Principle of peak suppression by reference kernel signal.

(4) if $i<i_{\max }$, the maximum iteration number, increase $i$ by one and return to step (2);

(5) terminate the iteration. The transmitted signal is obtained by: $x^{\prime}=x+\mathbf{c}^{(i)}$.

The overall process of the gradient iterative-based method is summarized in Figure 3.

\section{Proposed Pilot-Aided PAPR Reduction Method}

The conventional TR method uses dedicated pilots for PAPR reduction issue leading to a spectral efficiency loss. In this proposition, we use some of the scattered pilots dedicated to channel estimation for both PAPR reduction and channel estimation purposes. The main problem turns out then to find the pilot symbols at the transmitter which minimizes the PAPR value but also to find these pilot symbols at the receiver in order to achieve channel estimation.
That is, in order to achieve both operations, that is, PAPR reduction and channel estimation, the set $\mathbf{c}$ chosen for PAPR reduction has to undergo some particular laws known at the receiver. Figure 4 shows the general principle of the proposed method. In this scheme, the function $f(\cdot)$ reflects the particular law known at both the transmitter and receiver. In our proposition, we consider a simple multiplicative law given by

$$
\begin{gathered}
C_{k+1}=C_{k} \times \Omega \quad \forall k \in[0, \ldots, M-2] \text { or equivalently } \\
C_{k}=\Omega^{k} C_{0},
\end{gathered}
$$

where $\Omega$ denotes the step between two consecutive pilots and $C_{0}$ is the first pilot symbol.

The choice of $C_{k}$ and $\Omega$ could be either in continuous domain, that is, the pilots, could take any continuous value in $\mathbb{C}$, or they could take any discrete value from a discrete predefined set in discrete domain with a discrete value $\Omega$. Since the estimation of the pilots and $\Omega$ at the receiver in continuous domain could yield to a residual estimation error, and then to a slight system performance degradation, we propose to perform research in a discrete subset of $\mathbb{C}$. Equation (14) becomes

$$
\begin{gathered}
C_{0}=\lambda e^{j \phi}, \\
\Omega=e^{j \Delta}, \\
C_{k}=\lambda e^{j(\phi+k \Delta)},
\end{gathered}
$$

where $\lambda, \phi$, and $\Delta$ take values from a predefined set of discrete values. $\lambda$ is the boost factor applied to the dedicated subcarriers, $\phi$ is the initial phase value of the first pilot symbol, and $\Delta$ is the phase increment. The main reason to select a multiplicative law is due to simplicity issues and boost factor control, that is, power control of the transmitted sequences. Indeed, using (15), we can equivalently control the boost factor of all pilots (joint PAPR and channel estimation pilots). Again, we recall that the "discrete domain" means that instead of searching SOCP solution in $\mathbb{C}$, we 


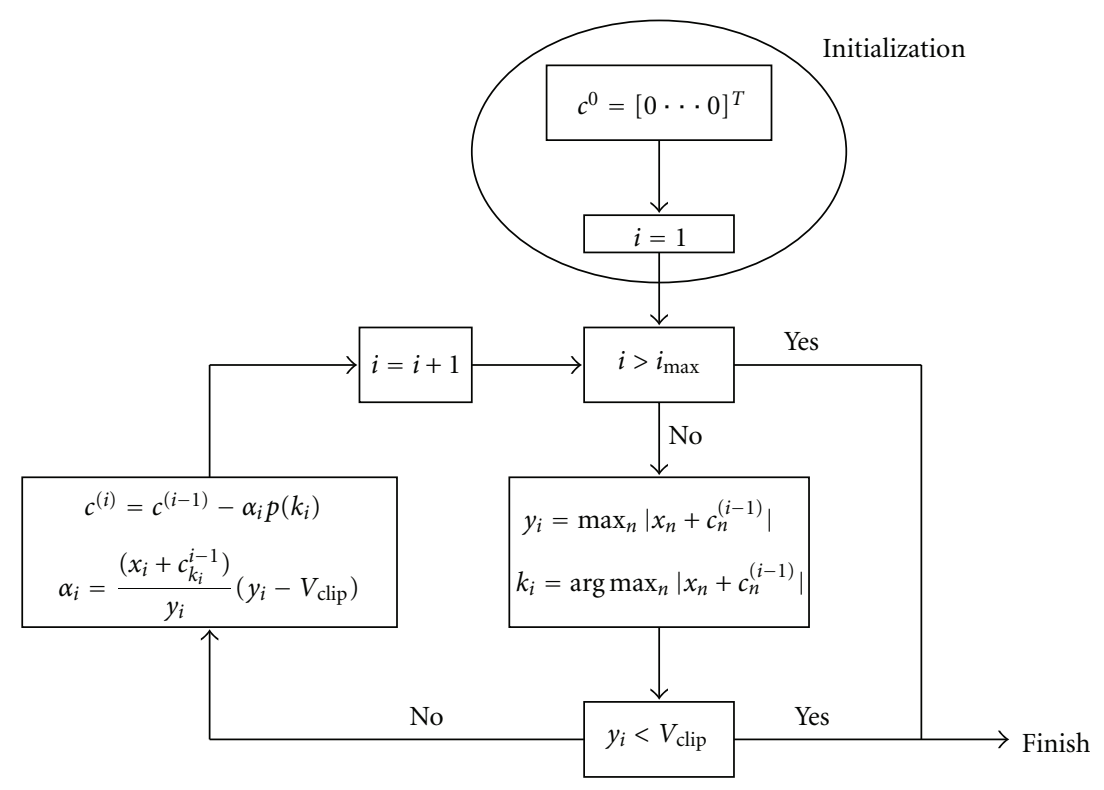

FIgURE 3: Principle of gradient iterative algorithm proposed in DVB-T2.

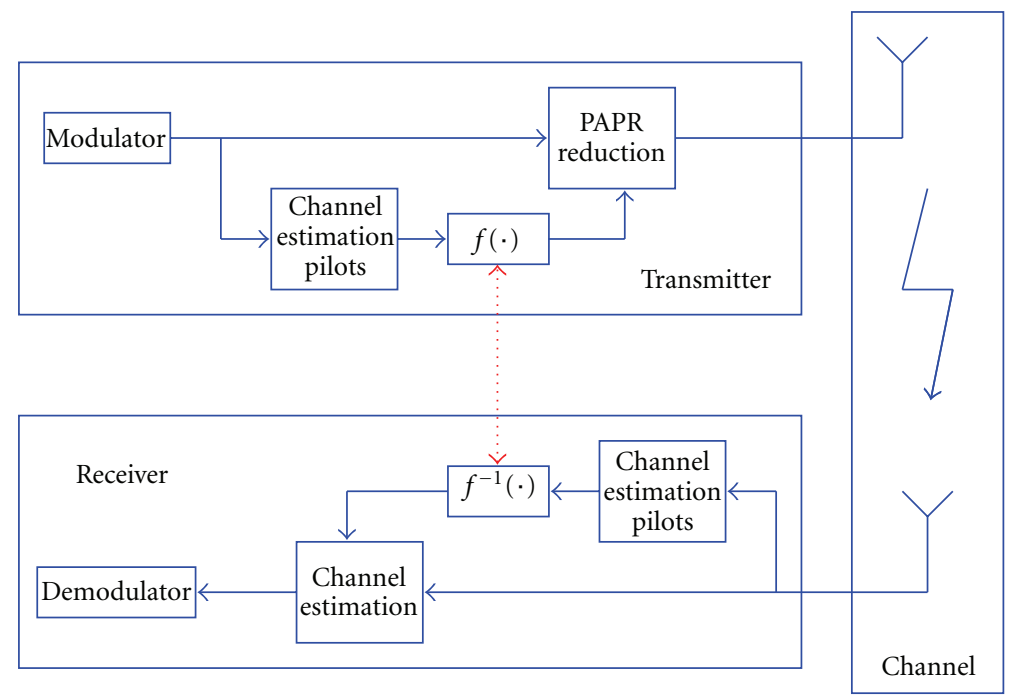

FIGURE 4: General principle of the proposed method.

search it in a predefined discrete set with a different discrete step for $\phi$ and $\Delta$, called $\mu(\phi)$, and $\mu(\Delta)$, respectively. Figure 5 shows the multiplicative law scheme and the evolution of the pilots from one index to another. $\lambda, \phi$, and $\Delta$ have to be selected in such a way to optimize the PAPR reduction gain and to limit channel estimation errors. This compromise will be detailed in next sections.

\section{Pilots Recovery and Channel Estimation}

In order to describe the channel estimation scheme and pilots recovery, frequency nonselective fading per subcarrier and time invariance during one OFDM symbol are assumed. Furthermore, the absence of intersymbol interference and intercarrier interference is guaranteed by the use of a guard interval longer than the maximum excess delay of the impulse response of the channel. In conventional OFDM schemes and under these assumptions, the received signal at the output of the FFT operation could be written as

$$
\mathbf{R}=\mathbf{H S}+\mathbf{W},
$$

where $\mathbf{H}$ denotes the $N \times N$ matrix which contains the complex channel coefficients, and $\mathbf{W}$ is the additive white Gaussian noise vector.

In conventional OFDM systems, the channel estimation is done by estimating the pilot channel coefficients $\hat{H}_{p}$ first and then by filtering the obtained coefficients using some conventional filters (the Wiener filter is widely used). The conventional OFDM channel estimation scheme is represented in Figure 6. Figure 7 shows the proposed channel 


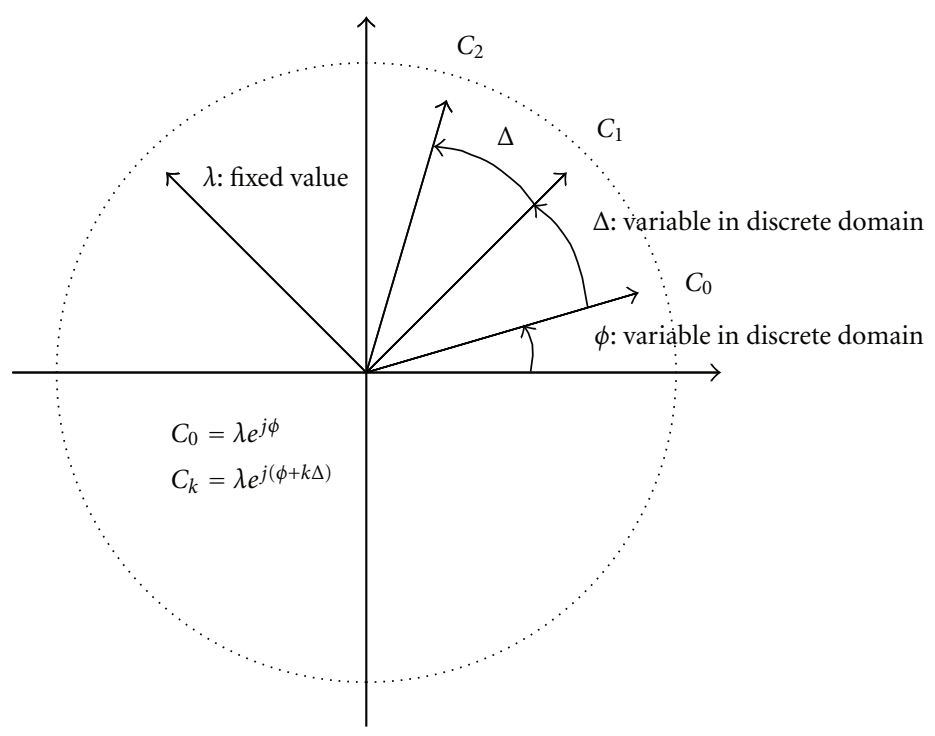

Figure 5: Multiplicative law scheme.

estimation scheme when some pilots are dedicated for PAPR issues. It is clear that there is a slight difference with the conventional estimation scheme shown in Figure 6. In our scheme, the pilot symbols $C_{p}$ used for PAPR reduction are not known a priori at the receiver. Therefore, the pilots' recovery and channel estimation procedure is performed in two steps. First, we determine the transmitted sequence $\widehat{C}_{p}$ used for PAPR reduction. In the second step, we determine the actual channel coefficients $\hat{H}_{k}$.

In order to accomplish the first step, we assume that the channel is almost constant between two successive OFDM symbols, that is, we assume that $\hat{\mathbf{H}}^{(l)} \approx \widehat{\mathbf{H}}^{(l-1)}$, where $l$ denotes the OFDM symbol index in time domain. Then, the pilot symbol used for PAPR reduction could be deduced by

$$
\bar{C}_{p}^{l}=\frac{R_{p}}{\widehat{H}_{p}^{(l-1)}} \quad \forall p \in P .
$$

By considering multiplicative law in discrete domain, received symbols at pilot positions are expressed as

$$
\bar{C}_{p}^{l}=\frac{H_{p}^{l} C_{p}^{l}+W_{p}^{l}}{\hat{H}_{k}^{(l-1)}} \approx C_{p}^{l}+\frac{W_{p}^{l}}{H_{p}^{l}} \quad \forall p \in P .
$$

By combining (18) with (15), we have

$$
\bar{C}_{p}^{l}=\lambda e^{j(\phi+p \Delta)}+\frac{W_{p}^{l}}{H_{p}^{l}} \quad \forall p \in P .
$$

Since $\mathbf{C}$ is in discrete frequency domain, the estimate, $\hat{C}_{p}^{l}$, of $C_{p}^{l}$ can be obtained from $\bar{C}_{p}^{l}$ by a simple quantization operation. As we consider that the boost factor $\lambda$ is known at the receiver, the set $\mathbf{C}$ is characterized by two unknown variables of $\phi$ and $\Delta$. Considering $\mu(\phi)$ and $\mu(\Delta)$ the elementary steps of $\phi$ and $\Delta$ in discrete domain, $\phi$ and $\Delta$ can be estimated as follows:

(i) estimation and decision of $\Delta$ :

$$
\begin{gathered}
\widehat{\Delta}=\operatorname{angle}\left[\sum_{p=1}^{M-1} \bar{C}_{p}^{l}\left(\bar{C}_{p-1}^{l}\right)^{*}\right], \\
\bar{\Delta}=D\left[\left.\hat{\Delta}\right|_{\mu(\Delta)}\right],
\end{gathered}
$$

where $D\left[\left.X\right|_{\alpha}\right]$ denotes decision function of $X$ in discrete domain with a step $\alpha$;

(ii) estimation and decision of $\phi$ :

$$
\begin{gathered}
\hat{\phi}=\operatorname{angle}\left[\sum_{p=0}^{M-1} \bar{C}_{p}^{l} e^{-j p \Delta}\right], \\
\bar{\phi}=D\left[\left.\hat{\phi}\right|_{\mu(\phi)}\right] .
\end{gathered}
$$

In order to evaluate our detection algorithm, we define the error detection probability (EDP) of $\phi$ and $\Delta$ as

$$
\begin{aligned}
& \operatorname{Pr}(\Delta)=\operatorname{Pr}\left\{|\bar{\Delta}-\Delta|>\frac{\mu(\Delta)}{2}\right\}, \\
& \operatorname{Pr}(\phi)=\operatorname{Pr}\left\{|\bar{\phi}-\phi|>\frac{\mu(\phi)}{2}\right\},
\end{aligned}
$$

where $\operatorname{Pr}\{|x|>\alpha\}$ denotes the probability that the absolute value of $x$ is greater than $\alpha$.

In the appendix, these probabilities are calculated in the case of AWGN channel. We obtain 


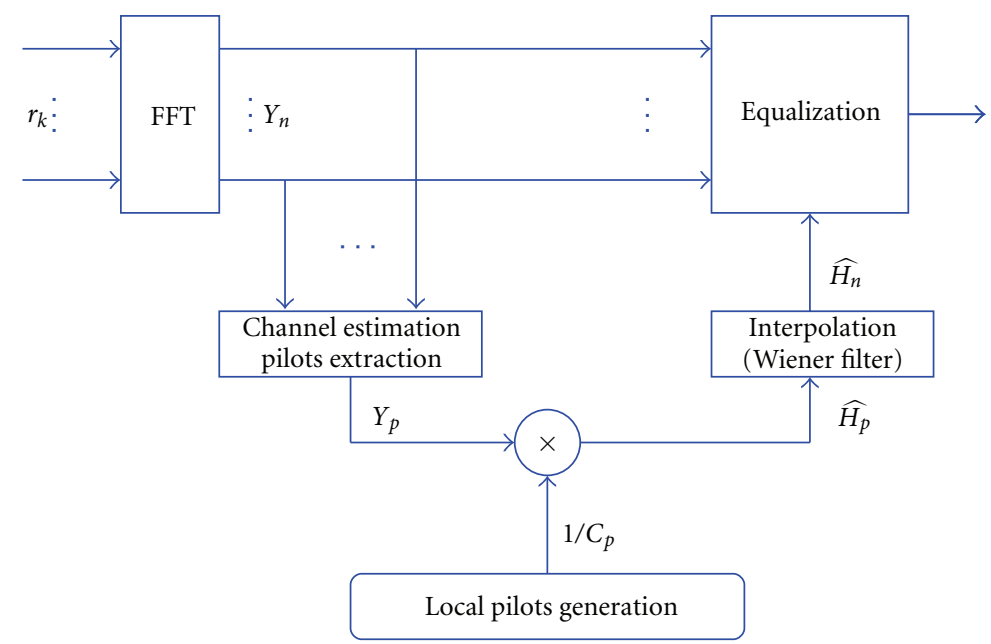

FIGURE 6: Conventional channel estimation scheme in OFDM systems.

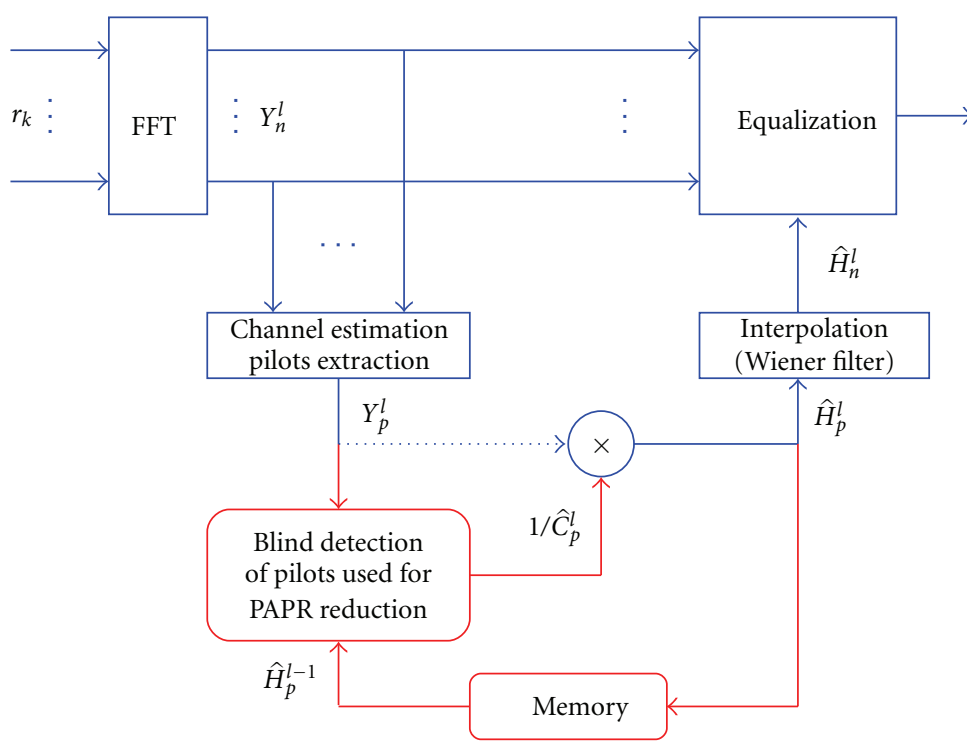

Figure 7: Modified channel estimation scheme.

$$
\begin{gathered}
\operatorname{Pr}(\Delta)=1-\operatorname{erf}\left(\frac{\mu(\Delta) / 2}{\sqrt{2\left(\sigma^{2} /(M-1)^{2} \lambda^{2}+2(M-1) \sigma^{4} /(M-1)^{2} \lambda^{4}\right)}}\right), \\
\operatorname{Pr}(\phi)=1-\operatorname{erf}\left(\frac{\mu(\phi) / 2}{\sqrt{\sigma^{2} / M \lambda^{2}}}\right) .
\end{gathered}
$$

The results of this computation are analyzed in the next section.

The estimation and detection of $\phi$ and $\Delta$ allow us to compute the transmitted pilot sequence. Once the transmitted pilot sequence is obtained, the second step consists of estimating the channel coefficients in the frequency domain. They could be obtained by the simple relationship

$$
\hat{H}_{k}^{l}=\frac{R_{k}}{\hat{C}_{k}^{l}} \quad \forall k \in P
$$




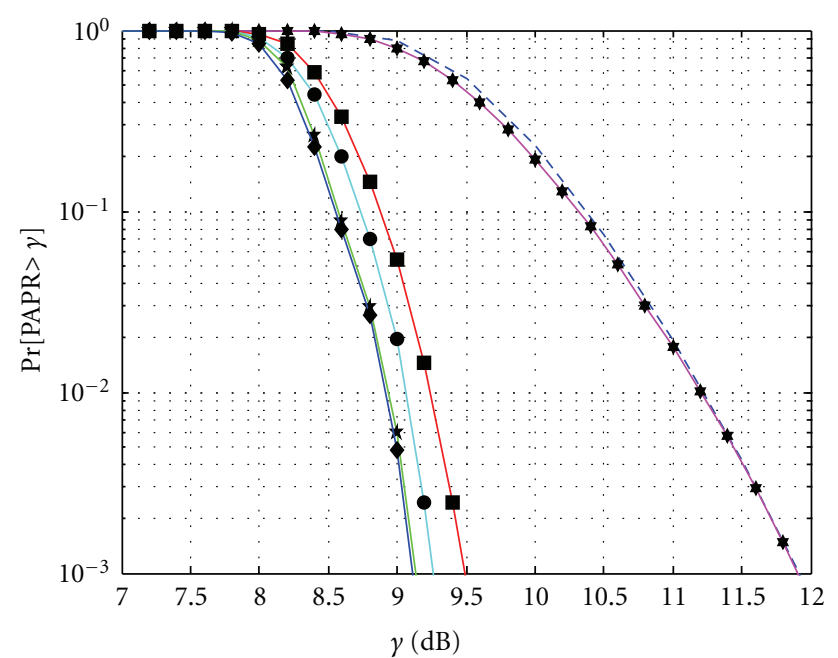

$---w / o$ PAPR reduction-theo. $\quad-$ Multiplicative law- $\mu=\pi / 4$

$\star w / o$ PAPR reduction-simul. $\star \star$ Multiplicative law- $\mu=\pi / 8$

$\neg$ Multiplicative law- $\mu=\pi / 2 \multimap$ Multiplicative law- $\mu=\pi / 16$

FIGURE 8: CCDF performance of multiplicative law using SOCP solution, continuous $\lambda, 16-\mathrm{QAM}$, and $2 \mathrm{k}$ mode.

Once the channel coefficients are computed on the pilot positions, the overall frequency channel response can be obtained by a simple Wiener filtering like in conventional channel estimation procedure. Both procedures are shown in Figures 6 and 7, respectively.

\section{Simulation Results and Discussion}

Simulation results are performed using DVB-T2 parameters. Some of the main parameters are summarized in Table 1. The PAPR parameters of the proposed method, that is, $\lambda, \Delta$, and $\phi$, are variable parameters which allow an optimization of both PAPR reduction and channel estimation processes. They will be specified in each simulation scenario.

Let us consider SOCP solution with a number of pilot tones $M$ equal to 8 . In fact, the SOCP solution is not possible using multiplicative law in continuous domain since the problem is not convex. In order to solve the problem, we consider discrete values of $\phi$ and $\Delta$ while maintaining $\lambda$ in continuous domain. More precisely, we consider $\lambda$ in continuous domain, and we change the values of $\phi$ from 0 to $2 \pi$ with a step of $\pi / 8$, while $\Delta$ varies from 0 to $2 \pi$ with variable step $\mu$, of $\pi / 2, \pi / 4, \pi / 8$, and $\pi / 16$. The results are presented in Figure 8 in terms of CCDF as declared in Section 2.

Figure 8 shows the CCDF of the multiplicative law using SOCP solution with respect to the continuous $\lambda$ value and different step values $\mu$. The PAPR gain increases when the step value $\mu$ decreases since the solution is approaching the continuous domain. Moreover, the solution converges when the step value $\mu$ reaches a limit value $\mu_{\text {lim }}=\pi / 8$. In other words, the PAPR gain increases when the pilots' values are optimized in continuous domain. The results obtained in Figure 8 show first that CCDF simulation results are close to theoretical value given in (6). Moreover, this figure shows that with a continuous $\lambda$ value, a phase discretization using a step $\mu=\pi / 8$ is enough to converge to the maximum PAPR reduction gain in the continuous domain.

6.1. Multiplicative Law Performance in Discrete Domain. We consider now the multiplicative law with $\phi$ and $\Delta$ in the discrete domain while $\lambda$ takes a value from a predefined set. Firstly, we present CCDF performance for a fixed $\lambda$ value and for $\phi$ and $\Delta$ varying from 0 to $2 \pi$ with a step of $\pi / 8$. The number of subcarriers used for PAPR reduction is always $M=8$. In this case, reducing PAPR is not aN SOCP problem any more but becomes a search of the optimum combination minimizing PAPR. At the receiver, pilots are recovered by a detection and estimation algorithm. Figure 9 gives a comparison between different PAPR reduction techniques, namely, SOCP solution, gradient technique, and our proposed technique with a predefined power value $\lambda=20 \mathrm{~dB}$. This figure shows that using our solution and this $\lambda$ value, we obtain slightly better results than in DVB-T2 gradient solution. However, the gradient solution requires an iterative complex implementation. Again, we recall that this method avoids the use of dedicated pilots for PAPR reduction issue, improving also spectral efficiency of the system (1\% in case of DVB-T2 specification).

In Figure 10, we present the PAPR gain in terms of CCDF, in comparison with the curve obtained without PAPR reduction technique, as a function of $\lambda$. We assume that $\phi$ and $\Delta$ change from 0 to $2 \pi$ with a step of $\pi / 8, \pi / 16$, and $\pi / 32$. This gain is evaluated at a CCDF $=10^{-3}$. It is also compared with the SOCP solution for multiplicative law ( $\lambda$ continuous). Figure 10 shows that the system performance is improved when the step of $\phi$ and $\Delta$ becomes smaller. This is because the number of pilot combinations increases when the step decreases. For each value of this step, there exists one optimum value of $\lambda$ for which the PAPR gain in terms of CCDF reaches the maximum.

Now, considering a discrete $\lambda$ value, we compare the performance of the proposed method, for a step of $\phi$ and $\Delta$ of $\pi / 8$, when $\lambda=20 \mathrm{~dB}$ which is the one maximizing the PAPR gain at $\mathrm{CCDF}=10^{-3}$ and when $\lambda$ is discrete, varying from $5 \mathrm{~dB}$ to $25 \mathrm{~dB}$ with a step of $3 \mathrm{~dB}$. The results are presented in Figure 11. For a CCDF $=10^{-3}$, the performance in terms of PAPR when $\lambda=20 \mathrm{~dB}$ is about $0.35 \mathrm{~dB}$ worse than the one of SOCP solution. It is about $0.17 \mathrm{~dB}$ worse than the case when $\lambda$ is discrete. In other words, we lose only $0.17 \mathrm{~dB}$ when a predefined value of $\lambda$ is selected instead of using a predefined set of discrete values.

In terms of complexity, the discrete solution is less complex than the SOCP solution (optimal solution). Indeed, our proposed PAPR reduction technique aims at searching the optimal solution in terms of $\lambda, \phi$, and $\Delta$ in a predefined discrete set of values while SOCP solution applies a search in continuous domain. Moreover, setting a predefined value of $\lambda$ yields to a reduced complexity implementation in comparison with the discrete $\lambda$ solution, however, with a $0.17 \mathrm{~dB}$ PAPR loss. On the other hand, in terms of 
TABLE 1: Simulation parameters, extracted from DVB-T2 standard.

\begin{tabular}{lc}
\hline Mode (OFDM size) & $2 \mathrm{~K}$ \\
\hline Number of subcarriers & $N_{\mathrm{FFT}}=2048$ \\
Guard interval length & $\mathrm{GI}=1 / 8$ \\
Modulation & $16-\mathrm{QAM}, 64-\mathrm{QAM}$ \\
Coding rate & $R=1 / 2$ \\
Oversampling factor & $L=4$ \\
Number of subcarriers used for PAPR reduction & $M=8,16,32$ \\
\hline
\end{tabular}

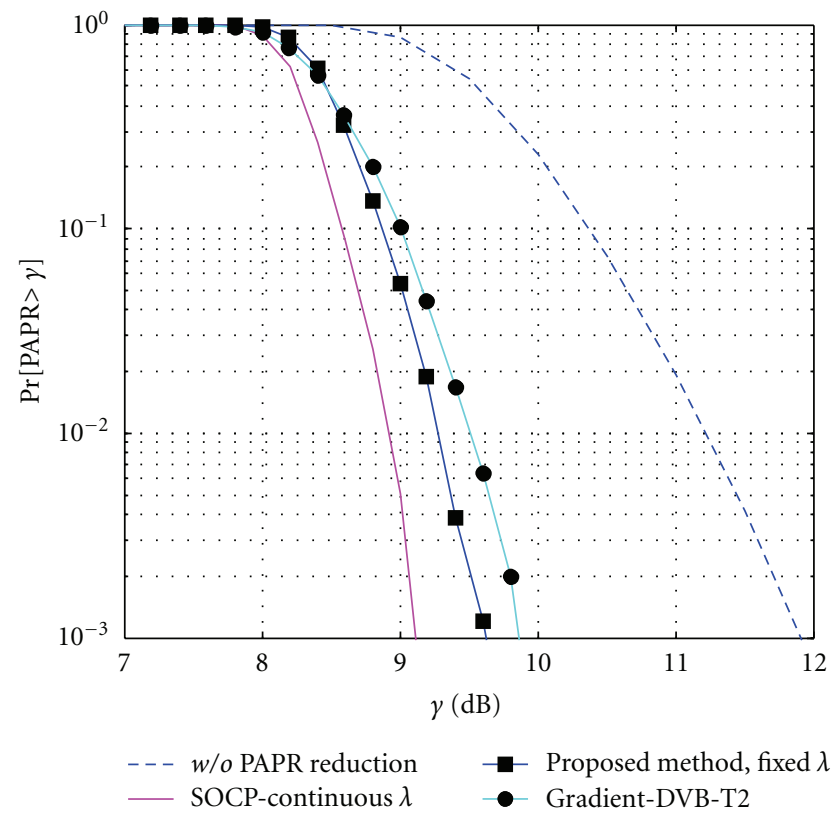

FIGURE 9: CCDF performance of multiplicative law in discrete domain, 16-QAM, $\lambda=20 \mathrm{~dB}$.

channel estimation, setting a predefined value of $\lambda$ implies an improved performance in comparison with the discrete case since we avoid quantization at the receiver. So, the proposed method with a predefined value of $\lambda$ presents a good tradeoff between channel estimation performance and PAPR gain.

In [4], OPS technique using Walsh-Hadamard sequences is chosen in order to make a blind detection of the transmitted sequence at the receiver side. In Figure 11, performance of this technique is given for WH sequences with length $N_{c}$ $=32$ chips. We can easily see that the performance of our proposed method is much better than the one proposed in [4]. A gain from $1.2 \mathrm{~dB}$ to $1.6 \mathrm{~dB}$ in terms of PAPR reduction is obtained in this case.

Finally, the performance in terms of PAPR gain is presented in Figure 12 for $M=8,16$, and 32 as a function of $\lambda$. Figure 12 shows that the PAPR gain performance slightly decreases when $M$ increases, but we note that the optimum value of $\lambda$ also decreases when $M$ increases. This is important because it means that we can reduce the pilot's power when increasing the number of subcarriers used for PAPR reduction.

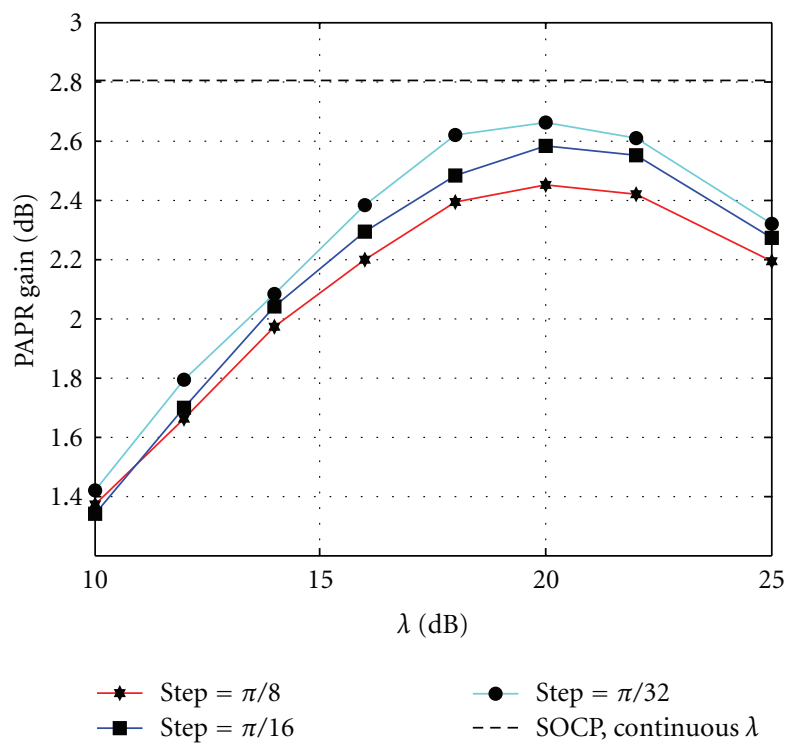

FIGURE 10: PAPR gain at a CCDF $=10^{-3}$ as a function of $\lambda, M=8$, 16-QAM.

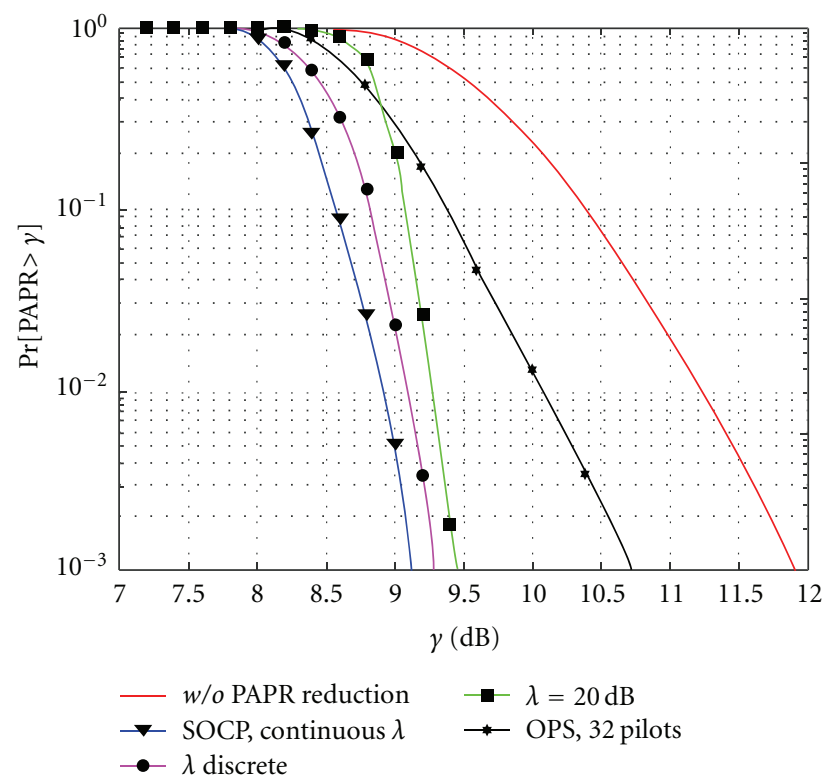

Figure 11: CCDF comparison in two cases: $\lambda=20 \mathrm{~dB}$ and discrete $\lambda$, with OPS technique [4], 16-QAM. 


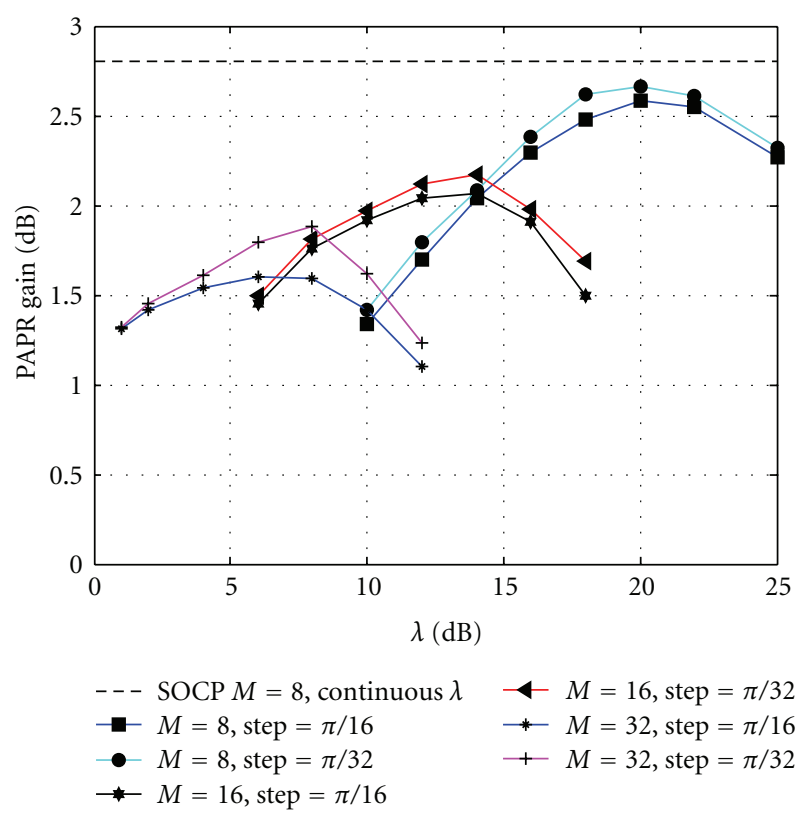

Figure 12: CCDF performance as a function of $\lambda$ and $M, 16-Q A M$.

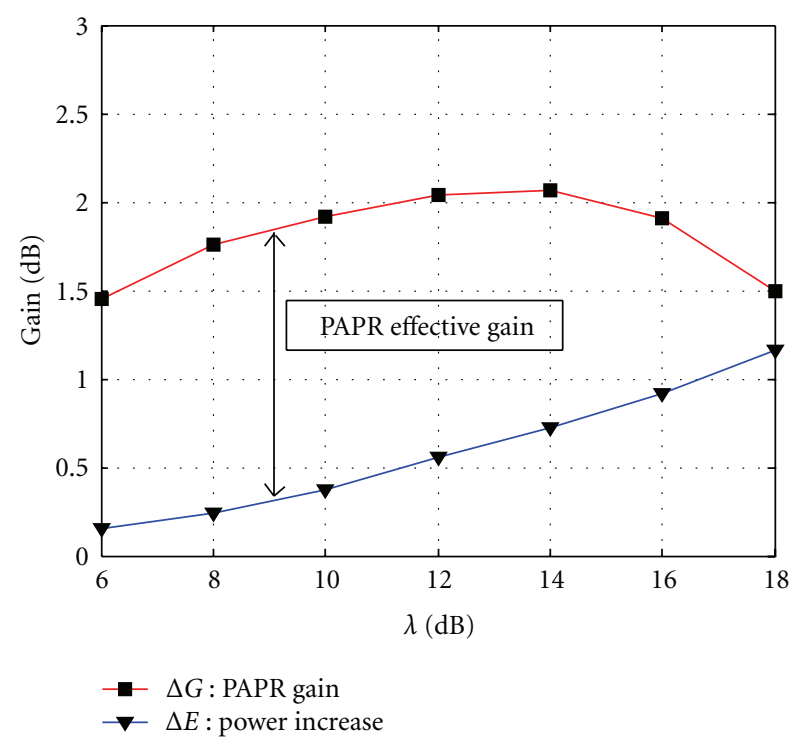

FIgURE 13: PAPR effective gain.

6.2. Comparison with DVB-T2 PAPR Techniques. First of all, one of the main advantages of this technique is that it could be used with rotated constellation schemes of DVB-T2 standard. This is not the case for ACE technique adopted in DVB-T2, where there is a restriction on rotated constellations. On the other hand, when the pilots are boosted by a factor $\lambda$, the transmitted power increases also. So, in order to have a fair comparison, we define the PAPR effective gain as the difference in $\mathrm{dB}$ between the PAPR gain $\Delta G$ and the power increase $\Delta E$ due to the use of boosted pilots (in both techniques), that is, $\mathrm{PAPR}_{\mathrm{eff}}=\Delta G-\Delta E$ (Figure 13).

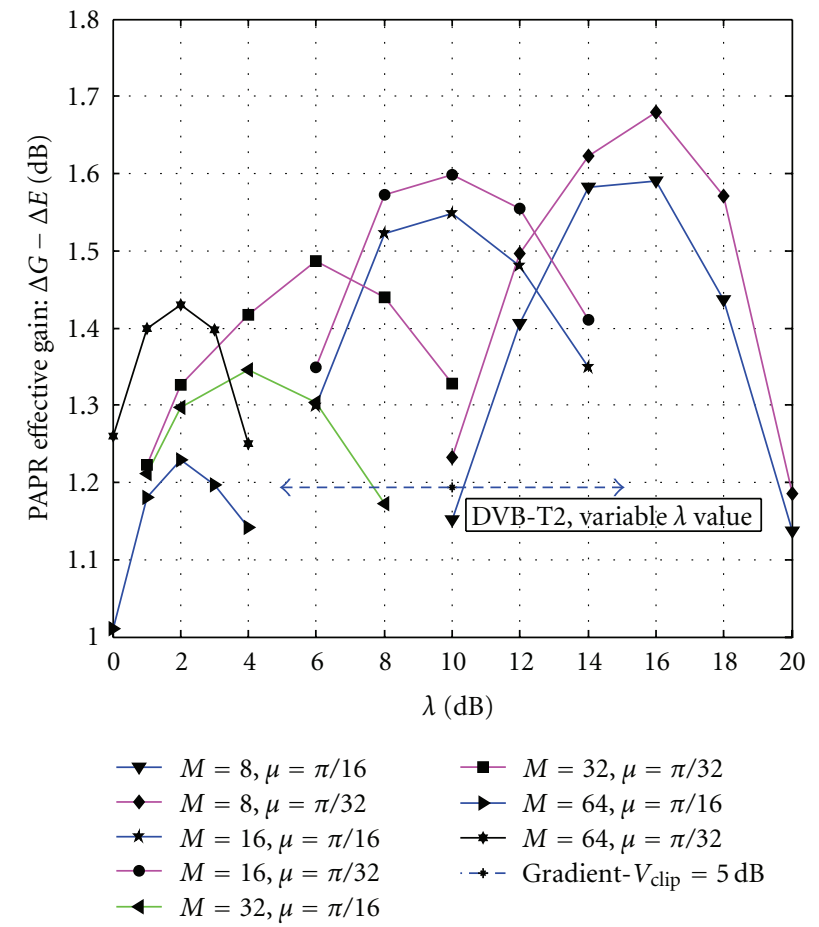

FIGURE 14: PAPR effective gain: comparison between proposed and DVB-T2 TR techniques, 16-QAM.

We evaluate in Figure 14 the PAPR effective gain of the proposed method as a function of the pilot power $\lambda$, which represents as previously the boost factor of the used pilots for different values of $M$, and of the elementary step $\mu$. We plot in the same figure the results obtained with the gradient-based method proposed in DVB-T2. We note that the gradient solution proposed in DVB-T2 does not specify the boost factor of each dedicated pilot but specifies the maximum permitted power of these pilots. Hence, the boost factor of these pilots in DVB-T2 could change from one symbol to another. In other words, the PAPR gain in gradient-based solution of DVB-T2 depends on a variable $\lambda$ value. The obtained results show that the performance of the proposed method is better than the one of the gradient-based method (TR technique) proposed in DVB-T2.

We also note that the optimal boost factor decreases when the number $M$ of channel estimation pilots used for PAPR reduction increases. In DVB-T2 standard, it is specified that the boost factor of the channel estimation pilots could take values between 2 and $7 \mathrm{~dB}$. Then, an optimal tradeoff should be found in terms of number of used pilots for PAPR reduction, PAPR effective gain, and channel estimation specifications. The same kind of results and conclusions could be given from Figure 15 for a 64-QAM constellation. In Figures 14 and 15, the performance of the DVB-T2 gradient algorithm is given as a reference result with a clipping value $V_{\text {clip }}=5 \mathrm{~dB}$. We note, however, that the gradient algorithm needs a smooth control of the transmitted powers of the PAPR pilots. Moreover, using the Gradient algorithm, the allocated powers are varying from one pilot to another. 


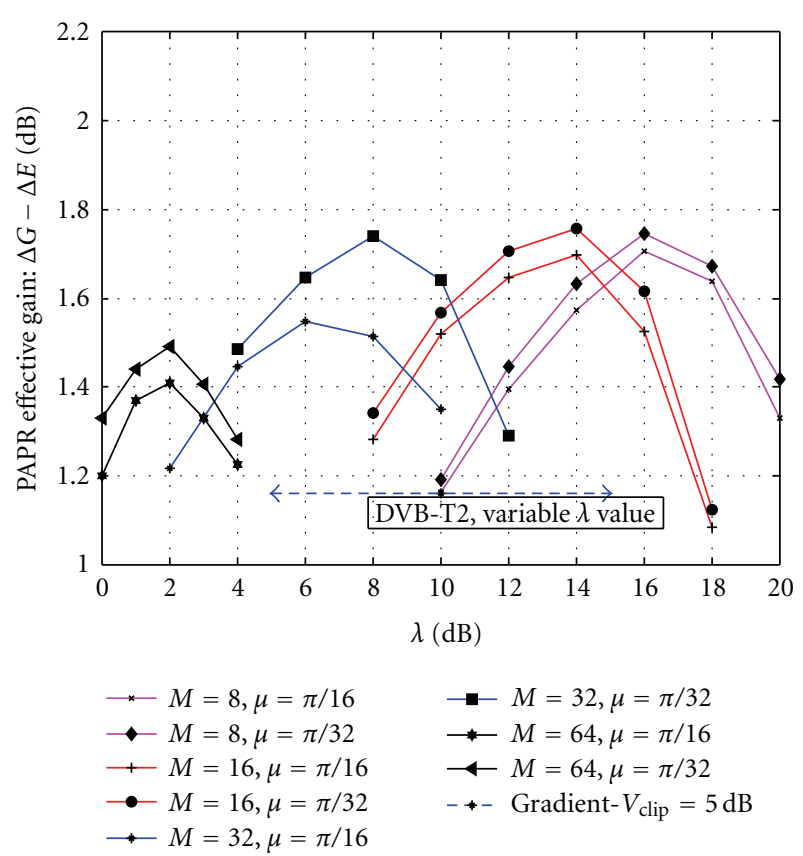

FIGURE 15: PAPR effective gain: comparison between proposed and DVB-T2 TR techniques, 64-QAM.

6.3. Channel Estimation Results. The goal of this section is to show how the system performance is affected by the use of dedicated channel estimation for both PAPR reduction and channel estimation issues.

In order to give more insights about the proposed technique, the error detection probability (EDP) of a sequence used for PAPR and channel estimation is first evaluated. Then, the mean square error (MSE) of channel estimation is performed with respect to the signal to noise ratio (SNR) of the system. The simulation results obtained hereafter are achieved at the output of the Wiener filter. The latter is a 1D filter applied in the frequency domain only. An improvement of the results could be obtained by filtering in $2 \mathrm{D}$, that is, frequency and time domain.

First, we verify the analytical performance evaluated in expressions (23). In order to evaluate the EDP values of $\Delta$ and $\phi$, we consider appropriated elementary step such as for each evaluation, EDP of the other parameter is negligible. For instance, we choose $\mu(\Delta)=\pi / 64$ and $\mu(\phi)=\pi / 4$ in order to evaluate the EDP of $\Delta ; \mu(\Delta)=\pi / 2$ and $\mu(\phi)=$ $\pi / 16$ in order to evaluate the EDP of $\phi$. Figure 16 illustrates the comparison between analytical and simulated EDPs for different SNR values. It shows that our theoretical analysis matches perfectly with simulations.

Figure 17 shows the EDP obtained by simulations of the pilot sequence at the receiver as a function of SNR for different values of the elementary steps $\mu(\phi)$ and $\mu(\Delta)$. This figure shows that it is better to quantify the phase difference between two successive pilot symbols with small quantifications steps $\mu(\Delta)$ than quantifying with small steps the possible initial phase value of the first pilot, that is, $\mu(\phi)$.

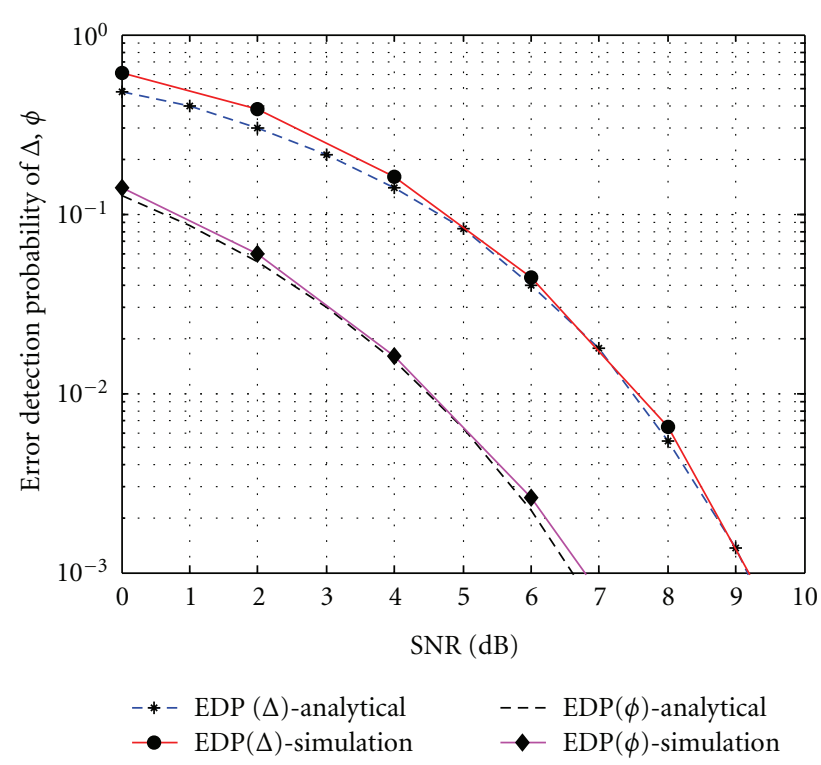

Figure 16: Analytical and simulated error detection probability.

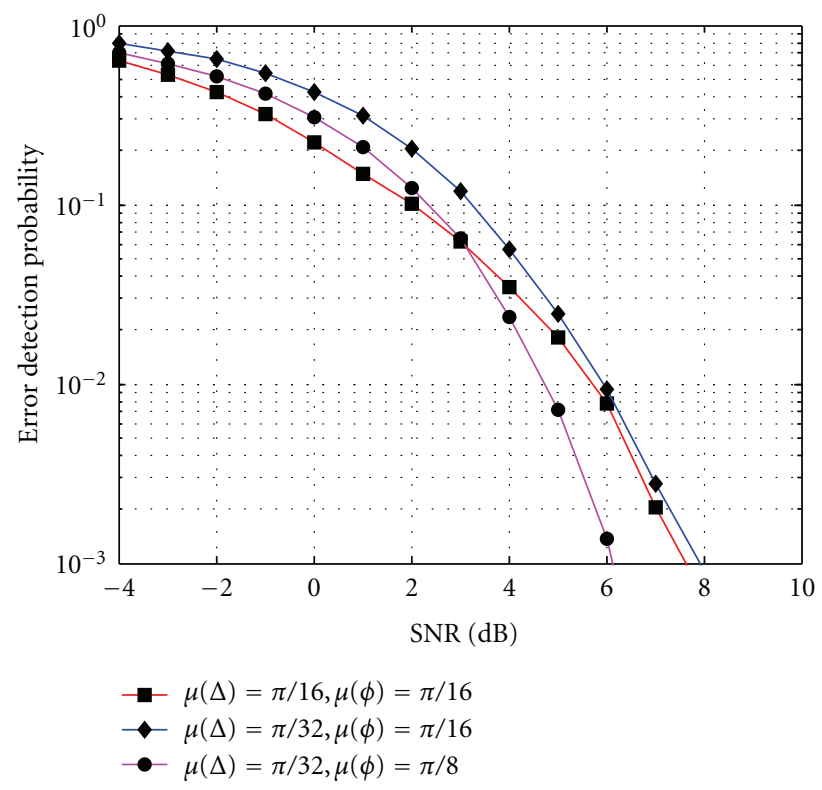

FIGURE 17: Error detection probability as a function of elementary steps.

In other words, it is more efficient to quantify more precisely $\Delta$ than the value of $\phi$.

Figure 18 presents the MSE of the channel coefficients estimation scheme using an F1 channel proposed in DVBT2 [1] using the conventional channel estimation scheme presented in DVB-T2 and the proposed channel estimation scheme. The conventional channel estimation relies on a traditional Wiener interpolation based on received channel estimation on pilot positions at the receiver. For the proposed method, as explained in Section 5, channel response on pilot positions used for PAPR reduction issue is recovered first by using channel response of the previous OFDM symbol. 


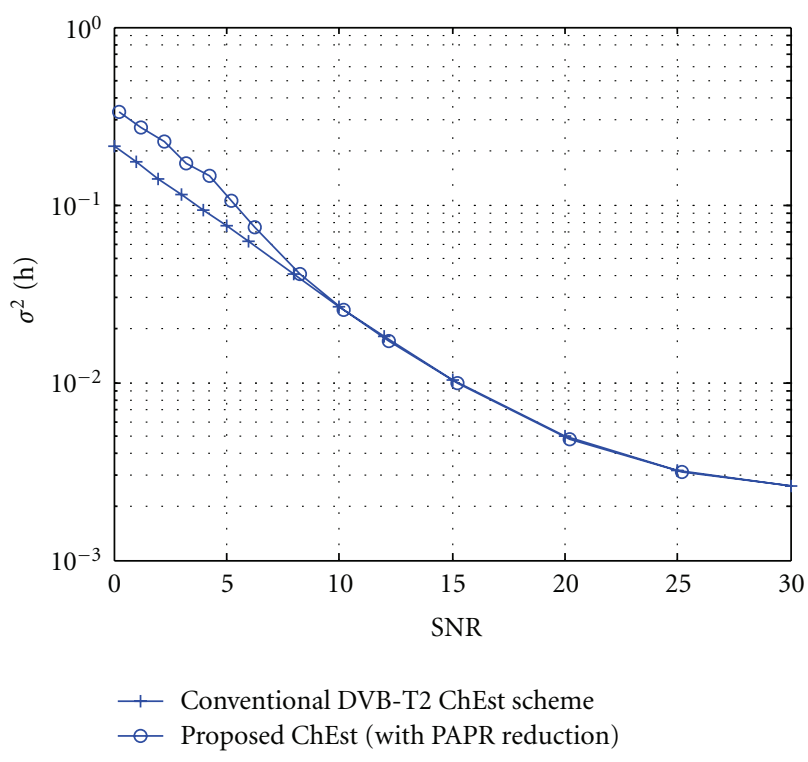

FIGURE 18: MSE of channel coefficients estimation, $2 \mathrm{~K}$ mode, 16QAM modulation, and F1 channel [1].

Then channel estimation on all subcarriers is performed using traditional Wiener interpolation. This figure shows that the MSE of the channel coefficients estimated using our method is negligible for an SNR value greater than $6 \mathrm{~dB}$. It is slightly higher than the conventional scheme for smaller SNR values. We recall that, for F1 channel and 16QAM constellation, the required SNR value to obtain a $\mathrm{BER}=10^{-7}$ at the output of the channel decoder is equal to $6.2 \mathrm{~dB}$ at a coding rate $R=1 / 2[1]$. The simulation results of this transmission scenario which is the most robust for a 16-QAM constellation are very important. Indeed, since the MSE is independent of the coding rate and, for higher coding rates the DVB-T2 requirements specify higher SNR values to make the system work properly, the proposed joint channel estimation scheme and PAPR reduction scheme will be always effective for these high SNR values.

We also evaluate the bit error rate (BER) of the overall DVB-T2 system. The F1 channel coefficients are estimated through dedicated pilots and then by applying a 2D Wiener filtering. Figure 19 shows the obtained BER performance using the conventional estimation scheme (Figure 6) and the proposed estimation scheme (Figure 7). As expected, the pilot sequence dedicated for channel estimation and used for PAPR reduction is well estimated at the receiver. Hence, the BER performance is not degraded.

Now, we consider a time-varying TU6 channel model given in [18]. The Doppler frequency $f_{d}$ is equal to $33 \mathrm{~Hz}$. Figure 20 gives the BER performance for a perfect channel estimation and a Wiener channel estimation without/with proposed PAPR reduction method. The obtained results show that the proposed scheme is efficient with high Doppler frequency scenario. We show in this figure that the overall degradation in comparison with perfect channel estimation is less than $1 \mathrm{~dB}$ where only $0.1 \mathrm{~dB}$ of SNR loss is due to the

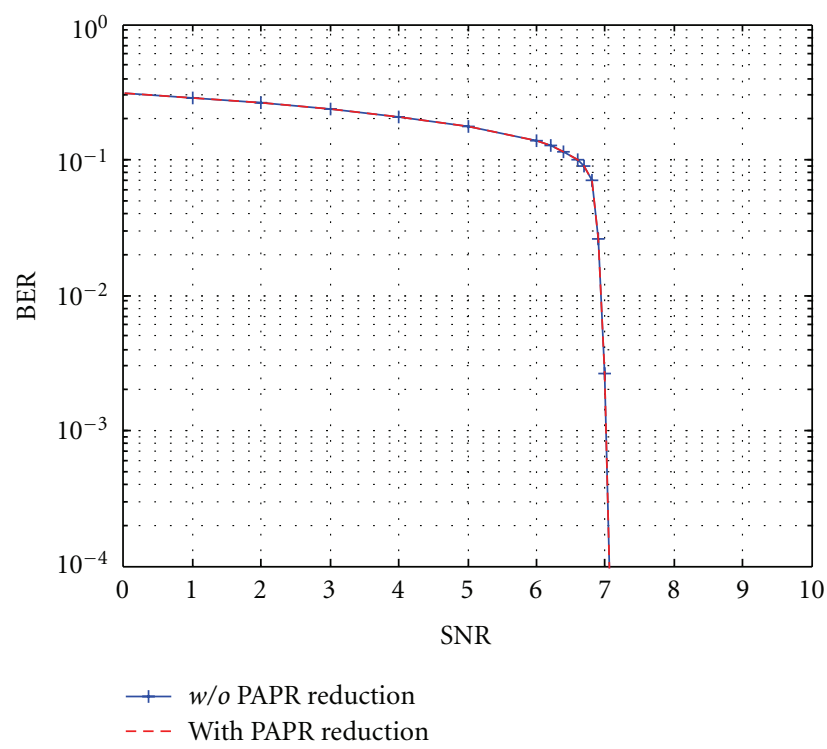

FIGURE 19: BER performance, $2 \mathrm{~K}$ mode, 16-QAM modulation, and F1 channel.

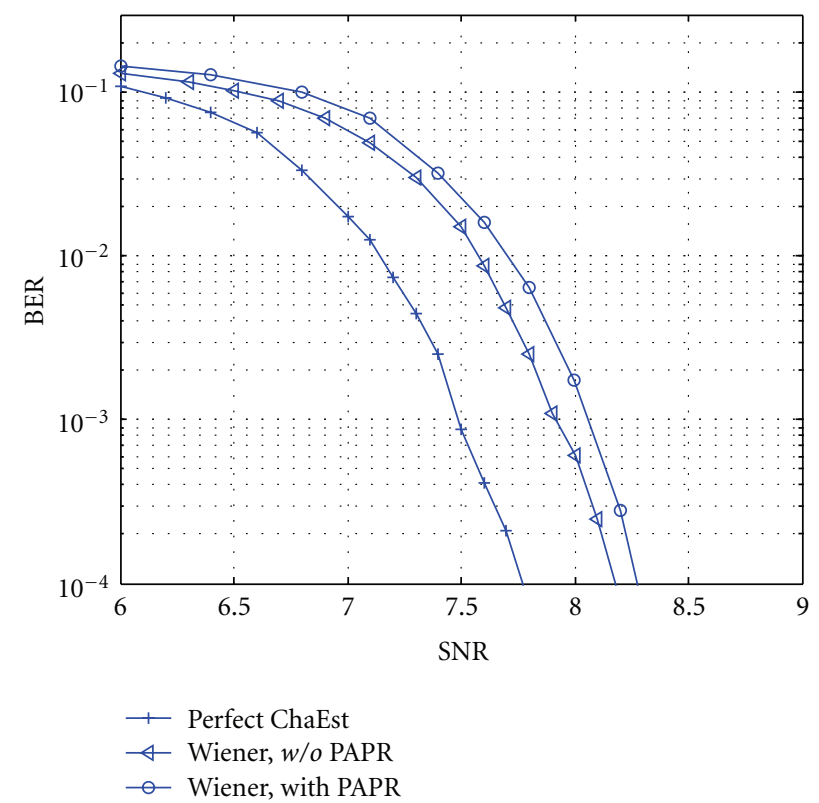

FIGURE 20: BER performance, $2 \mathrm{~K}$ mode, 16-QAM modulation, and TU6 channel.

joint application of PAPR reduction and channel estimation scheme.

6.4. Results Summary. Based on the conclusions given in previous sections, this section summarizes optimal parameters and the corresponding PAPR effective gains using the proposed technique. The different results previously presented have been obtained in the $2 \mathrm{k}$ mode. The same analysis has been done in the $8 \mathrm{k}$ mode. Tables 2 and 3 summarize the main parameters chosen as a good tradeoff between PAPR gain, channel estimation performance, and 
TABLE 2: Optimal parameters using the proposed technique, $2 \mathrm{~K}$ mode.

\begin{tabular}{lcccrr}
\hline Modulation & Number of pilots: PP2 & $M$ & Elementary steps of $\phi$ and $\Delta$ & $\lambda_{\text {opt }}$ & PAPR effective gain \\
\hline \multirow{2}{*}{ 16-QAM } & 143 & 32 & $\mu(\Delta)=\pi / 32, \mu(\phi)=\pi / 8$ & $5 \mathrm{~dB}$ & $1.49 \mathrm{~dB}$ \\
& 143 & & DVB-T2 $\left(V_{\text {clip }}=5 \mathrm{~dB}-18\right.$ pilots $)$ & $1.19 \mathrm{~dB}$ \\
64-QAM & 143 & 32 & $\mu(\Delta)=\pi / 64, \mu(\phi)=\pi / 16$ & $7 \mathrm{~dB}$ & $1.80 \mathrm{~dB}$ \\
& 143 & & DVB-T2 $\left(V_{\text {clip }}=5 \mathrm{~dB}-18\right.$ pilots $)$ & & $1.16 \mathrm{~dB}$ \\
\hline
\end{tabular}

TABLE 3: Optimal parameters using the proposed technique, $8 \mathrm{~K}$ mode.

\begin{tabular}{lccccc}
\hline Modulation & Number of pilots: PP2 & $M$ & Elementary steps of $\phi$ and $\Delta$ & $\lambda_{\text {opt }}$ & PAPR effective gain \\
\hline \multirow{2}{*}{ 16-QAM } & 569 & 128 & $\mu(\Delta)=\pi / 64, \mu(\phi)=\pi / 16$ & $5 \mathrm{~dB}$ & $1.47 \mathrm{~dB}$ \\
& 569 & & DVB-T2 $\left(V_{\text {clip }}=5 \mathrm{~dB}-72\right.$ pilots $)$ & $1.39 \mathrm{~dB}$ \\
64-QAM & 569 & 128 & $\mu(\Delta)=\pi / 128, \mu(\phi)=\pi / 32$ & $5 \mathrm{~dB}$ & $1.48 \mathrm{~dB}$ \\
& 569 & & DVB-T2 $\left(V_{\text {clip }}=5 \mathrm{~dB}-72\right.$ pilots $)$ & & $1.38 \mathrm{~dB}$ \\
\hline
\end{tabular}

optimal transmitted power in the $2 \mathrm{k}$ and $8 \mathrm{k}$ modes for 16 QAM and 64-QAM modulations. In all cases, the proposed technique presents better PAPR gain than the DVB-T2 TR technique, which requires less transmitted power on pilot symbols (the maximum value of the boost factor $\lambda$ of the PAPR pilots in DVB-T2 is equal to $10 \mathrm{~dB}$ ) but also a spectral efficiency increase of $1 \%$ when it is compared with the actual DVB-T2 standard. It could be also applied using the rotated constellation adopted in DVB-T2. We should note that these parameters, that is, $M, \lambda, \Delta$, and $\phi$, are applicable to the context of DVB-T2. However, they should be optimized to other contexts especially when the system parameters like the number of subcarriers and the constellation size change. Nevertheless, the optimization process remains unchanged.

\section{Conclusion}

A novel PAPR reduction method based on channel estimation pilots is addressed in this paper. By using channel estimation pilots to reduce PAPR value, dedicated pilots for PAPR reduction purpose are avoided improving the spectral efficiency of the system. These pilots have to be related by a particular law in order to allow their detection at the receiver side. Multiplicative law in discrete frequency domain is then investigated. Simulations, using the new DVB-T2 standard chain, showed that with appropriate parameters, the proposed method can achieve up to $1.80 \mathrm{~dB}$ in terms of PAPR effective gain. In comparison with the gradient-based method initially proposed in DVB-T2, the proposed method presents better performance in terms of PAPR reduction while avoiding the use of dedicated pilots. As a consequence, it allows achieving $1 \%$ of spectral efficiency gain. At the receiver side, only a slight modification is required. Simulations have shown that no degradation is caused by this additional function. The obtained results demonstrate the relevance of this method for future broadcasting of OFDM-based systems.

\section{Appendix}

This section aims to compute the error detection probability of the pilot sequence. It involves the computation of the error detection probability of $\Delta$ and $\phi$ in a Gaussian channel.

\section{A. Error Detection Probability of $\Delta$}

From (20), we have

$$
\begin{gathered}
\widehat{\Delta}=\text { angle }\left[\sum_{p=1}^{M-1} \bar{C}_{p}^{l}\left(\bar{C}_{p-1}^{l}\right)^{*}\right], \\
\bar{C}_{p}^{l}=\lambda e^{j(\phi+p \Delta)}+\frac{W_{p}^{l}}{H_{p}^{l}} \quad \forall p \in P .
\end{gathered}
$$

Since we assume a Gaussian channel, received pilots can be expressed as

$$
\bar{C}_{p}^{l}=\lambda e^{j(\phi+p \Delta)}+n_{p} \quad \forall p \in P,
$$

where $n_{p}$ denotes complex additive white gaussian noise (AWGN), at the pilot position $p$, of zero mean and of standard deviation $\sigma$, noted $\mathbf{N}\left(0, \sigma^{2}\right)$. It can be decomposed as follows: $n_{p}=n_{p, R}+j n_{p, I}$ where $n_{p, R}$ and $n_{p, I}$ denote real and imaginary parts of the complex noise $n_{p}$ which are AWGN of zero mean and with standard deviation $\sigma / \sqrt{2}$. 
Equation (A.1) becomes

$$
\begin{gathered}
\hat{\Delta}=\operatorname{angle}\left[\sum_{p=1}^{M-1}\left(\lambda e^{j(\phi+p \Delta)}+n_{p}\right)\left(\lambda e^{j(\phi+(p-1) \Delta)}+n_{p-1}\right)^{*}\right] \\
\hat{\Delta}=\operatorname{angle}\left[(M-1) \lambda^{2} e^{j \Delta}+\sum_{p=1}^{M-1}\left(\lambda e^{j(\phi+p \Delta)} n_{p-1}^{*}+\lambda e^{-j(\phi+(p-1) \Delta)} n_{p}+n_{p} n_{p-1}^{*}\right)\right] \\
\widehat{\Delta}=\operatorname{angle}\left[(M-1) \lambda^{2} e^{j \Delta}\left(1+\frac{e^{-j \Delta}}{(M-1) \lambda^{2}} \sum_{p=1}^{M-1}\left(\lambda e^{j(\phi+p \Delta)} n_{p-1}^{*}+\lambda e^{-j(\phi+(p-1) \Delta)} n_{p}+n_{p} n_{p-1}^{*}\right)\right)\right] .
\end{gathered}
$$

Then, we have

$$
\begin{gathered}
\widehat{\Delta}-\Delta=\operatorname{angle}\left[1+\frac{1}{(M-1) \lambda^{2}} \sum_{p=1}^{M-1}\left(\lambda e^{j(\phi+(p-1) \Delta)} n_{p-1}^{*}+\lambda e^{-j(\phi+p \Delta)} n_{p}+n_{p} n_{p-1}^{*}\right)\right], \\
\widehat{\Delta}-\Delta=\operatorname{angle}[1+\underbrace{\frac{1}{(M-1) \lambda} \sum_{p=1}^{M-1}\left(e^{j(\phi+(p-1) \Delta)} n_{p-1}^{*}+e^{-j(\phi+p \Delta)} n_{p}\right)}_{A}+\underbrace{\frac{1}{(M-1) \lambda^{2}} \sum_{p=1}^{M-1}\left(n_{p} n_{p-1}^{*}\right)}_{B}] .
\end{gathered}
$$

By substituting $n_{p}=n_{p, R}+j n_{p, I}$ in (A.5), the sum $A$ becomes

$$
\begin{aligned}
& A=\frac{1}{(M-1) \lambda} \sum_{p=1}^{M-1}\left(e^{j(\phi+(p-1) \Delta)} n_{p-1}^{*}+e^{-j(\phi+p \Delta)} n_{p}\right)=\frac{1}{(M-1) \lambda}\left[\sum_{p=0}^{M-2} e^{j(\phi+p \Delta)} n_{p}^{*}+\sum_{p=1}^{M-1} e^{-j(\phi+p \Delta)} n_{p}\right] \\
& A=\frac{1}{(M-1) \lambda}\left[e^{j \phi} n_{0}^{*}+e^{-j(\phi+(M-1) \Delta)} n_{M-1}+\sum_{p=1}^{M-2}\left(e^{j(\phi+p \Delta)} n_{p}^{*}+e^{-j(\phi+p \Delta)} n_{p}\right)\right] \\
& A=\frac{1}{(M-1) \lambda}\left\{\begin{array}{c}
\underbrace{\left[\cos (\phi) n_{0, R}+\sin (\phi) n_{0, I}+\cos [\phi+(M-1) \Delta] n_{0, R}+\sin [\phi+(M-1) \Delta] n_{0, I}\right]}_{A 1} \\
+j \underbrace{\left[\sin (\phi) n_{0, R}-\cos (\phi) n_{0, I}+\cos [\phi+(M-1) \Delta] n_{0, I}-\sin [\phi+(M-1) \Delta] n_{0, R}\right]}_{A 2}
\end{array}\right. \\
& +\underbrace{2 \sum_{p=1}^{M-2}\left(\cos (\phi+p \Delta) n_{p, R}+\sin (\phi+p \Delta) n_{p, I}\right)}_{A 3} .
\end{aligned}
$$


Property 1. Let $\left\{X_{k}\right\}_{k=1 \cdots n}$ be the set of $n$ AWGN variables of $\mathbf{N}\left(0, \sigma_{k}^{2}\right)$ and $\left\{a_{k}\right\}_{k=1 \cdots n}$ be the set of $n$ constants, then we have [19]

$$
\sum_{k=1}^{n} a_{k} X_{k} \longmapsto N\left(0, \sum_{k=1}^{n} a_{k}^{2} \sigma_{k}^{2}\right) .
$$

According to Property 1 , knowing that $n_{p, R}$ and $n_{p, I}$ are uncorrelated and of $\mathbf{N}\left(0, \sigma^{2} / 2\right)$ for all $k=1 \cdots n$, we deduce

$$
\begin{gathered}
A_{1} \longmapsto N\left(0, \sigma^{2}\right), \\
A_{2} \longmapsto N\left(0, \sigma^{2}\right), \\
A_{3} \longmapsto N\left(0,2(M-2) \sigma^{2}\right) .
\end{gathered}
$$

So we have

$$
A \longmapsto N\left(0, \frac{(2 M-3) \sigma^{2}}{(M-1)^{2} \lambda^{2}}\right)+j N\left(0, \frac{\sigma^{2}}{(M-1)^{2} \lambda^{2}}\right) .
$$

Similarly, the sum $B$ of (A.6) can be expressed as

$$
\begin{aligned}
& B=\frac{1}{(M-1) \lambda^{2}} \sum_{p=1}^{M-1}\left(n_{p} n_{p-1}^{*}\right) \\
& =\frac{1}{(M-1) \lambda^{2}} \sum_{p=1}^{M-1}\left(n_{p, R}+j n_{p, I}\right)\left(n_{p-1, R}-j n_{p-1, I}\right), \\
& B=\frac{1}{(M-1) \lambda^{2}} \sum_{p=1}^{M-1}\left(n_{p, R} n_{p-1, R}+n_{p, I} n_{p-1, I}\right) \\
& +j\left(n_{p, I} n_{p-1, R}-n_{p, R} n_{p-1, I}\right), \\
& B=\frac{1}{(M-1) \lambda^{2}} \\
& \times[\underbrace{\sum_{p=1}^{M-1}\left(n_{p, R} n_{p-1, R}+n_{p, I} n_{p-1, I}\right)}_{B 1} \\
& +\underbrace{j \sum_{p=1}^{M-1}\left(n_{p, I} n_{p-1, R}-n_{p, R} n_{p-1, I}\right)}_{B 2}] .
\end{aligned}
$$

Property 2. Let $X_{1}$ and $X_{2}$ be two independent AWGN variables of $\mathbf{N}\left(0, \sigma^{2}\right)$ and $X=X_{1} X_{2}$, then we have

$$
\begin{aligned}
& E[X]=E\left[X_{1} X_{2}\right]=E\left[X_{1}\right] E\left[X_{2}\right]=0, \\
& E\left[X^{2}\right]=E\left[\left(X_{1} X_{2}\right)^{2}\right]=E\left[\left(X_{1}\right)^{2}\right] E\left[\left(X_{2}\right)^{2}\right]=\sigma^{4} .
\end{aligned}
$$

So variance of $X$ is

$$
V[X]=E\left[(X-E[X])^{2}\right]=E\left[X^{2}\right]=\sigma^{4} .
$$

According to Property 2, the products $n_{p, R} n_{p-1, R}$, $n_{p, I} n_{p-1, I}, n_{p, R} n_{p-1, I}, n_{p, I} n_{p-1, R}$ follow the same law with zero mean and with standard deviation $\sigma^{2}$. According to the central limit theory, $B_{1}$ and $B_{2}$ are Gaussian with zero mean and a variance $2(M-1) \sigma^{4}$. So, we have

$$
B \longmapsto N\left(0, \frac{2(M-1) \sigma^{4}}{(M-1)^{2} \lambda^{4}}\right)+j N\left(0, \frac{2(M-1) \sigma^{4}}{(M-1)^{2} \lambda^{4}}\right) .
$$

By substituting (A.10) and (A.13) in (A.5), equation (A.5) becomes

$$
\begin{aligned}
& \widehat{\Delta}-\Delta \\
&=\operatorname{angle}\left[1+N\left(0, \frac{(2 M-3) \sigma^{2}}{(M-1)^{2} \lambda^{2}}\right)+j N\left(0, \frac{\sigma^{2}}{(M-1)^{2} \lambda^{2}}\right)\right. \\
&\left.+N\left(0, \frac{2(M-1) \sigma^{4}}{(M-1)^{2} \lambda^{4}}\right)+j N\left(0, \frac{2(M-1) \sigma^{4}}{(M-1)^{2} \lambda^{4}}\right)\right],
\end{aligned}
$$

$$
\widehat{\Delta}-\Delta=\operatorname{angle}[\underbrace{1+N\left(0, \frac{(2 M-3) \sigma^{2}}{(M-1)^{2} \lambda^{2}}+\frac{2(M-1) \sigma^{4}}{(M-1)^{2} \lambda^{4}}\right)+j N\left(0, \frac{\sigma^{2}}{(M-1)^{2} \lambda^{2}}+\frac{2(M-1) \sigma^{4}}{(M-1)^{2} \lambda^{4}}\right)}_{Z}] .
$$

Assuming a large SNR, real part of $Z$ can be approximated to 1 and its imaginary part can be approximated to
0 , equation (A.15) can be approximated by using angle $(X) \approx \operatorname{Im}(X)$ : 


$$
\widehat{\Delta}-\Delta \longmapsto N\left(0, \frac{\sigma^{2}}{(M-1)^{2} \lambda^{2}}+\frac{2(M-1) \sigma^{4}}{(M-1)^{2} \lambda^{4}}\right) .
$$

So, $\widehat{\Delta}-\Delta$ can be expressed as a Gaussian noise of zero mean with variance $\sigma^{2} /(M-1)^{2} \lambda^{2}+2(M-1) \sigma^{4} /(M-1)^{2} \lambda^{4}$.

Assuming $\mu(\Delta)$ the elementary step of $\Delta$, the error detection probability is given by

$$
\begin{aligned}
\operatorname{Pr}(\Delta)= & \operatorname{Pr}\left(|\widehat{\Delta}-\Delta|>\frac{\mu(\Delta)}{2}\right) \\
= & 1-\operatorname{Pr}\left(|\hat{\Delta}-\Delta|<\frac{\mu(\Delta)}{2}\right) \\
= & 1-\operatorname{Pr}\left(\widehat{\Delta}-\Delta<\frac{\mu(\Delta)}{2}\right) \\
& +\operatorname{Pr}\left(\hat{\Delta}-\Delta<\frac{-\mu(\Delta)}{2}\right) \\
\operatorname{Pr}(\Delta)= & 1-\operatorname{Pr}\left(\widehat{\Delta}-\Delta<\frac{\mu(\Delta)}{2}\right) \\
& +\left[1-\operatorname{Pr}\left(\widehat{\Delta}-\Delta<\frac{\mu(\Delta)}{2}\right)\right] \\
= & 2\left[1-\operatorname{Pr}\left(\widehat{\Delta}-\Delta<\frac{\mu(\Delta)}{2}\right)\right] .
\end{aligned}
$$

Property 3. Let $X$ be an AWGN variable of $\mathbf{N}\left(\mu, \sigma^{2}\right)$, then we have

$$
\operatorname{Pr}(X<\alpha)=\frac{1}{2}\left[1+\operatorname{erf}\left(\frac{\alpha-\mu}{\sigma \sqrt{2}}\right)\right]
$$

Finally, according to Property 3, we obtain

$$
\begin{aligned}
\operatorname{Pr}(\Delta) & =1-\operatorname{erf} \\
& \times\left(\frac{\mu(\Delta) / 2}{\sqrt{2\left(\sigma^{2} /(M-1)^{2} \lambda^{2}+2(M-1) \sigma^{4} /(M-1)^{2} \lambda^{4}\right)}}\right) .
\end{aligned}
$$

\section{B. Error Detection Probability of $\phi$}

To compute the error detection probability of $\phi$, we assume that $\Delta$ is well estimated, then we have

$$
\begin{gathered}
\hat{\phi}=\operatorname{angle}\left[\sum_{p=0}^{M-1} \bar{C}_{p}^{l} e^{-j p \Delta}\right] \\
=\operatorname{angle}\left[\sum_{p=0}^{M-1}\left(\lambda e^{j(\phi+p \Delta)}+n_{p}\right) e^{-j p \Delta}\right], \\
\hat{\phi}=\operatorname{angle}\left[\sum_{p=0}^{M-1} \lambda e^{j \phi}+n_{p} e^{-j p \Delta}\right] \\
=\operatorname{angle}\left[M \lambda e^{j \phi}+\sum_{p=0}^{M-1} n_{p} e^{-j p \Delta}\right], \\
\hat{\phi}=\operatorname{angle}\left[M \lambda e^{j \phi}\left(1+\frac{1}{M \lambda} \sum_{p=0}^{M-1} n_{p} e^{-j p \Delta}\right)\right] .
\end{gathered}
$$

We deduce

$$
\begin{aligned}
\hat{\phi}-\phi & =\operatorname{angle}\left[1+\frac{1}{M \lambda} \sum_{p=0}^{M-1} n_{p} e^{-j p \Delta}\right] \\
& =\operatorname{angle}\left[1+\frac{1}{M \lambda} \sum_{p=0}^{M-1}\left(n_{p, R}+n_{p, I}\right)\right.
\end{aligned}
$$

$$
\times(\cos (p \Delta)-j \sin (p \Delta))]
$$

It can be approximated by using angle $(X) \approx \operatorname{Im}(X)$

$$
\widehat{\phi}-\phi \approx \frac{1}{M \lambda} \sum_{p=0}^{M-1}\left[n_{p, I} \cos (p \Delta)-n_{p, R} \sin (p \Delta)\right] .
$$

According to the central limit theory, we deduce,

$$
\widehat{\phi}-\phi \longmapsto N\left(0, \frac{\sigma^{2}}{2 M \lambda^{2}}\right)
$$


Assuming $\mu(\phi)$ the elementary step of $\phi$, the error detection probability of $\phi$ is given by

$$
\begin{aligned}
\operatorname{Pr}(\phi)= & \operatorname{Pr}\left(|\hat{\phi}-\phi|>\frac{\mu(\phi)}{2}\right) \\
= & 1-\operatorname{Pr}\left(|\hat{\phi}-\phi|<\frac{\mu(\phi)}{2}\right) \\
= & 1-\operatorname{Pr}\left(\hat{\phi}-\phi<\frac{\mu(\phi)}{2}\right) \\
& +\operatorname{Pr}\left(\hat{\phi}-\phi<\frac{-\mu(\phi)}{2}\right) \\
\operatorname{Pr}(\phi)= & 1-\operatorname{Pr}\left(\hat{\phi}-\phi<\frac{\mu(\phi)}{2}\right) \\
& +\left[1-\operatorname{Pr}\left(\hat{\phi}-\phi<\frac{\mu(\phi)}{2}\right)\right] \\
= & 2\left[1-\operatorname{Pr}\left(\hat{\phi}-\phi<\frac{\mu(\phi)}{2}\right)\right]
\end{aligned}
$$

According to Property 3, we obtain

$$
\operatorname{Pr}(\phi)=1-\operatorname{erf}\left(\frac{\mu(\phi) / 2}{\sqrt{\sigma^{2} / M \lambda^{2}}}\right) .
$$

\section{Acknowledgment}

The authors would like to thank the CELTIC ENGINES project for its support of this work.

\section{References}

[1] DVB, "Frame structure channel coding and modulation for a second generation digital terrestrial television broadcasting system (DVB-T2),” ETSI EN 302755 V1.1.1 September 2009.

[2] U. Reimers, "Digital video broadcasting," IEEE Communications Magazine, vol. 36, no. 6, pp. 104-110, 1998.

[3] R. Van Nee and R. Prasad, OFDM for Wireless Multimedia Communications, Artech House, Boston, Mass, USA, 2000.

[4] M. J. F. G. García, O. Edfors, and J. M. Páez-Borrallo, "Peak power reduction for OFDM systems with orthogonal pilot sequences," IEEE Transactions on Wireless Communications, vol. 5, no. 1, pp. 47-51, 2006.

[5] S. H. Han and J. H. Lee, "An overview of peak-to-average power ratio reduction techniques for multicarrier transmission," IEEE Wireless Communications, vol. 12, no. 2, pp. 56-65, 2005.

[6] X. Li and L. J. Cimini, "Effects of clipping and filtering on the performance of OFDM," in Proceedings of the 47th IEEE Vehicular Technology Conference (VTC '97), vol. 3, pp. 16341638, Phoenix, Ariz, USA, May 1997.

[7] A. E. Jones, T. A. Wilkinson, and S. K. Barton, "Block coding scheme for reduction of peak to mean envelope power ratio of multicarrier transmission schemes," Electronics Letters, vol. 30, no. 25, pp. 2098-2099, 1994.

[8] S. H. Müller and J. B. Huber, "OFDM with reduced peakto-average power ratio by optimum combination of partial transmit sequences," Electronics Letters, vol. 33, no. 5, pp. 368369, 1997.

[9] R. W. Bäuml, R. F. H. Fischer, and J. B. Huber, "Reducing the peak-to-average power ratio of multicarrier modulation by selected mapping," Electronics Letters, vol. 32, no. 22, pp. 2056-2057, 1996.

[10] A. D. S. Jayalath and C. Tellambura, "Reducing the peak-toaverage power ratio of orthogonal frequency division multiplexing signal through bit or symbol interleaving," Electronics Letters, vol. 36, no. 13, pp. 1161-1163, 2000.

[11] B. S. Krongold and D. L. Jones, "PAR reduction in OFDM via active constellation extension," IEEE Transactions on Broadcasting, vol. 49, no. 3, pp. 258-268, 2003.

[12] J. Tellado-Mourelo, Peak-to-average power ratio reduction for multicarrier Modulation, Ph.D. thesis, Stanford University, September 1999.

[13] I. M. Mahafeno, Y. Louët, and J. F. Hélard, "Peak-to-average power ratio reduction using second order cone programming based tone reservation for terrestrial digital video broadcasting systems," IET Communications, vol. 3, no. 7, pp. 1250-1261, 2009.

[14] A. Aggarwal and T. H. Meng, "Minimizing the peak-to-average power ratio of OFDM signals using convex optimization," IEEE Transactions on Signal Processing, vol. 54, no. 8, pp. 30993110, 2006.

[15] S. Zabre, J. Palicot, Y. Louët, and C. Lereau, "SOCP approach for OFDM peak-to-average power ratio reduction in the signal adding context," in Proceedings of the 6th IEEE International Symposium on Signal Processing and Information Technology (ISSPIT '07), pp. 834-839, Vancouver, Canada, August 2007.

[16] M. Sharif, M. Gharavi-Alkhansari, and B. H. Khalaj, "On the peak-to-average power of OFDM signals based on oversampling," IEEE Transactions on Communications, vol. 51, no. 1, pp. 72-78, 2003.

[17] R. van Nee and A. de Wild, "Reducing the peak-to-average power ratio of OFDM," in Proceedings of the 48th IEEE Vehicular Technology Conference (VTC '98), vol. 3, pp. 2072 2076, Ottawa, Canada, May 1998.

[18] DVB, "Implementation guidelines for a second generation digital terrestrial television broadcasting system (DVB-T2)," ETSI TR 102831 V0.9.17 November 2009.

[19] M. K. Simon, Probability Distributions Involving Gaussian Random Variables, Springer, New York, NY, USA, 2006. 

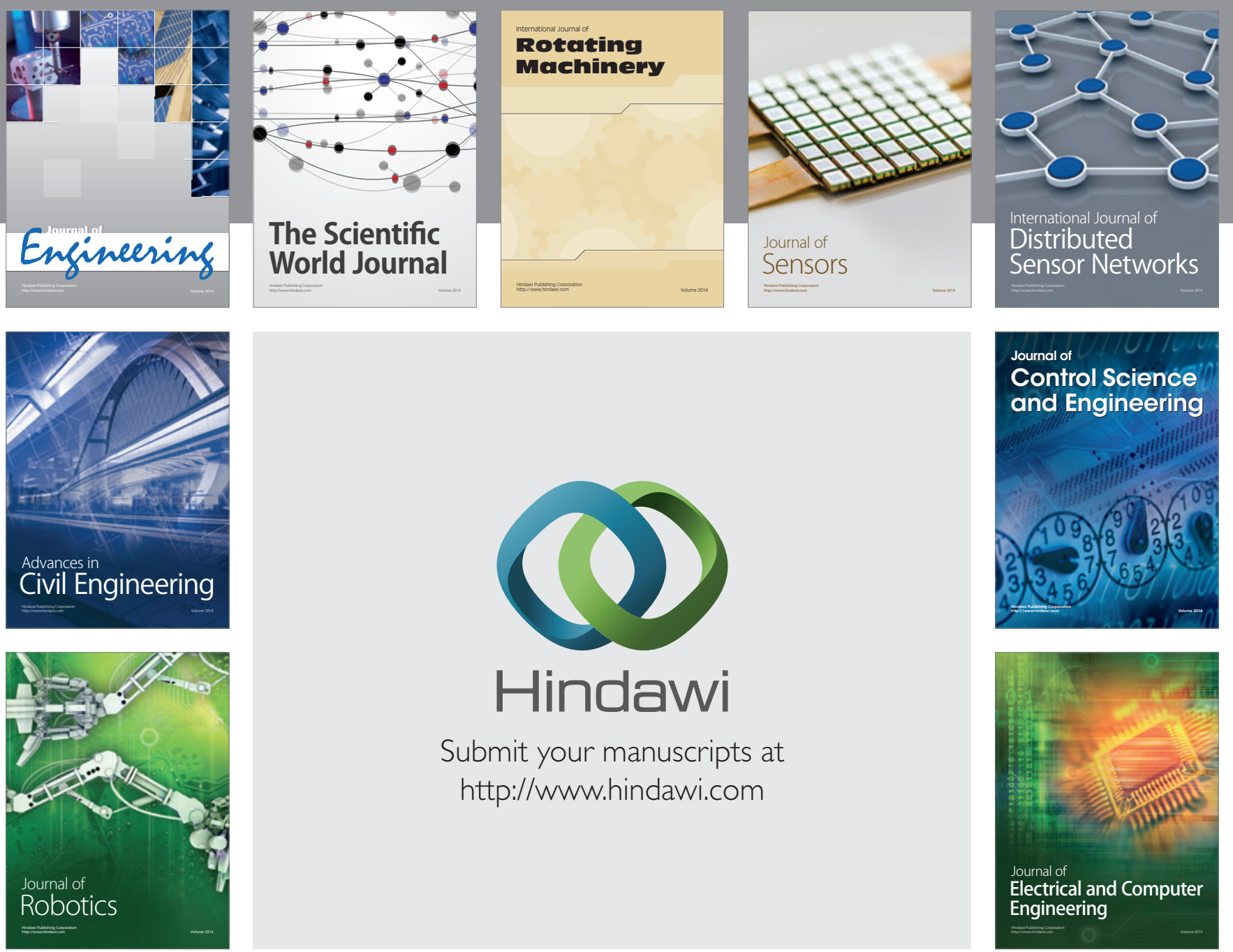

Submit your manuscripts at

http://www.hindawi.com
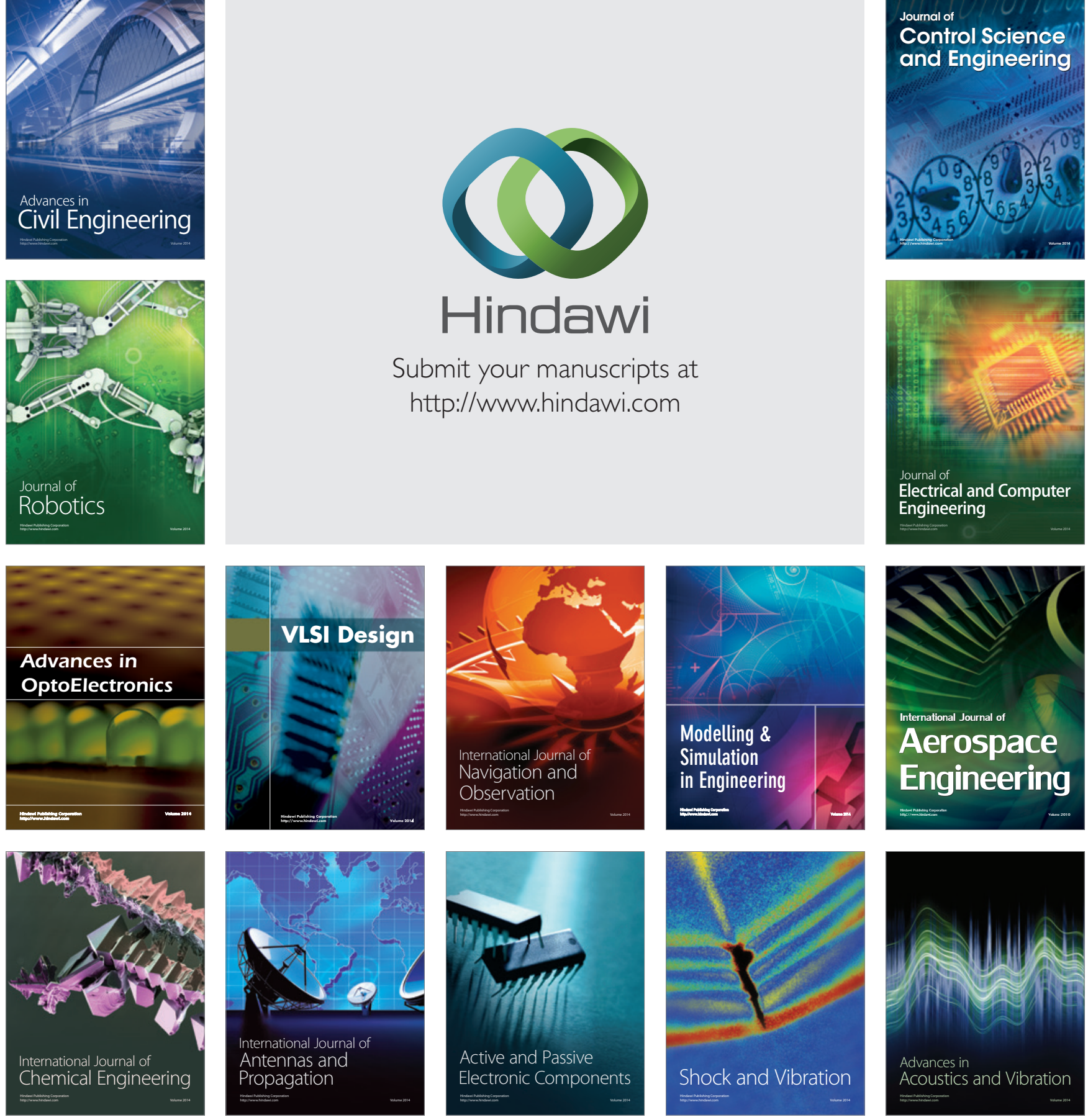